\title{
Crosstalk between cancer-associated fibroblasts and immune cells in the tumor microenvironment: new findings and future perspectives
}

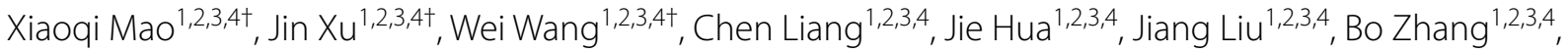
Qingcai Meng ${ }^{1,2,3,4^{*}}$, Xianjun $\mathrm{Yu}^{1,2,3,4^{*}}$ and Si Shi ${ }^{1,2,3,4^{*}}$ (1)

\begin{abstract}
Cancer-associated fibroblasts (CAFs), a stromal cell population with cell-of-origin, phenotypic and functional heterogeneity, are the most essential components of the tumor microenvironment (TME). Through multiple pathways, activated CAFs can promote tumor growth, angiogenesis, invasion and metastasis, along with extracellular matrix (ECM) remodeling and even chemoresistance. Numerous previous studies have confirmed the critical role of the interaction between CAFs and tumor cells in tumorigenesis and development. However, recently, the mutual effects of CAFs and the tumor immune microenvironment (TIME) have been identified as another key factor in promoting tumor progression. The TIME mainly consists of distinct immune cell populations in tumor islets and is highly associated with the antitumor immunological state in the TME. CAFs interact with tumor-infiltrating immune cells as well as other immune components within the TIME via the secretion of various cytokines, growth factors, chemokines, exosomes and other effector molecules, consequently shaping an immunosuppressive TME that enables cancer cells to evade surveillance of the immune system. In-depth studies of CAFs and immune microenvironment interactions, particularly the complicated mechanisms connecting CAFs with immune cells, might provide novel strategies for subsequent targeted immunotherapies. Herein, we shed light on recent advances regarding the direct and indirect crosstalk between CAFs and infiltrating immune cells and further summarize the possible immunoinhibitory mechanisms induced by CAFs in the TME. In addition, we present current related CAF-targeting immunotherapies and briefly describe some future perspectives on CAF research in the end.
\end{abstract}

Keywords: Cancer-associated fibroblasts, Tumor-infiltrating immune cells, Tumor microenvironment, Tumor immune microenvironment, Cell-cell interaction, Immune suppression, CAF-targeted therapy, Cancer

*Correspondence: mengqingcai@fudanpci.org; yuxianjun@fudanpci.org; shisi@fudanpci.org

${ }^{\dagger}$ Xiaoqi Mao, Jin Xu and Wei Wang contributed equally to this work and shared the first authorship.

${ }^{1}$ Department of Pancreatic Surgery, Fudan University Shanghai Cancer Center, No. 270 Dong'An Road, Xuhui District, Shanghai 200032, China

Full list of author information is available at the end of the article

\section{Introduction}

In recent years, the tumor microenvironment (TME) has received increasing attention due to its crucial roles in tumor immune suppression, distant metastasis, local resistance and the targeted therapy response [1-4]. The TME is a highly complicated system mainly composed of tumor cells, infiltrating immune cells (such as macrophages, dendritic cells and lymphocytes), cancer-associated stromal cells (such as cancer-associated fibroblasts original author(s) and the source, provide a link to the Creative Commons licence, and indicate if changes were made. The images or other third party material in this article are included in the article's Creative Commons licence, unless indicated otherwise in a credit line to the material. If material is not included in the article's Creative Commons licence and your intended use is not permitted by statutory regulation or exceeds the permitted use, you will need to obtain permission directly from the copyright holder. To view a copy of this licence, visit http://creativecommons.org/licenses/by/4.0/. The Creative Commons Public Domain Dedication waiver (http://creativeco mmons.org/publicdomain/zero/1.0/) applies to the data made available in this article, unless otherwise stated in a credit line to the data. 
$(\mathrm{CAFs})$ ), endothelial cells and lipocytes, along with the extracellular matrix (ECM) and multiple signaling molecules $[5,6]$. As one of the most important stromal components in the TME, CAFs have shown biological heterogeneity in many aspects, including the cell of origin, phenotype and function [7,8]. Originating from a variety of cell types, CAFs are characterized by increased expression of markers such as alpha smooth muscle actin $(\alpha-S M A)$, fibroblast activation protein (FAP), fibroblastspecific protein 1 (FSP1), platelet-derived growth factor receptor (PDGFR) $\alpha / \beta$ and vimentin [9]. Most of CAF subpopulations usually exhibit cancer-promoting effects, while the discovery of cancer-restraining CAFs (rCAFs), which are reported to exert inhibitory effects on tumor progression, indicates that some subsets are just the opposite [10]. Substantial previous reports have demonstrated that CAFs participate in multiple stages of tumor development through diverse pathways [11-13]. Through bidirectional signaling with tumor cells and other cells mediated by CAF-derived cytokines, chemokines, growth factors and exosomes within the TME, CAFs not only facilitate tumor proliferation but also induce immune evasion of cancer cells [14-16]. Moreover, CAFs are also able to degrade stromal ECM by releasing matrix metalloproteinases (MMPs) while synthesizing new matrix proteins to provide structural support for tumor invasion and angiogenesis $[17,18]$. Overall, more specific roles and detailed mechanisms of CAFs in cancer pathogenesis and progression remain to be further explored.

The tumor immune microenvironment (TIME) is a novel proposed concept that has been reported to be closely related to the clinical prognosis of patients with tumors [19]. Distinct immune cell populations, including innate and adaptive immune cells, such as myeloid cells and lymphocytes within the TME, comprise most of the TIME $[20,21]$. Notably, the TIME also determines the state of the immune response in the TME, which primarily depends on the composition and activity of infiltrated immune cells, as well as several correlated influencing factors, including the cell surface expression of immune checkpoint molecules and alterations in the associated matrix [20]. Currently, an increasing number of researchers have begun to focus on the immunosuppressive effect of CAFs that is achieved by interactions with TIME components, especially immune cells [22-24]. For instance, CAFs are capable of restricting the recruitment of immune effector cells such as CD8 $+\mathrm{T}$ cells into tumor tissues through the secretion of different chemokines [25]. Moreover, the proportions of immunosuppressive cells such as M2-type macrophages, regulatory T (Treg) cells and myeloid-derived suppressor cells (MDSCs), which are modified by CAFs, have been shown to be significantly increased in the TIME, thereby contributing to tumor immune suppression [26-28]. Additionally, some cytokines secreted by activated immune cells, such as interleukin (IL)-1 $\beta$, can induce the transformation of normal fibroblasts into proinflammatory CAFs and further facilitate the recruitment of inhibitory immune cells and immune suppression in the TME [29]. Undeniably, a deep understanding of the multidimensional interactions between CAFs and infiltrating immune cells within the TME will help us better to determine the immunosuppressive mechanisms induced by CAFs, and further exploration of these interactions will probably identify more potential molecular targets for CAF-targeted therapy.

This review mainly focuses on recent advances in the crosstalk between CAFs and tumor-infiltrating immune cells, immune checkpoint molecules and related ECM alterations in the TME, along with the possible mechanisms of CAF-induced immune suppression according to these interactions. We also describe the current understanding of the origins, activators, heterogeneity and plasticity of CAFs. Finally, we introduce major CAFbased targeted immunotherapeutic strategies that may enhance antitumor immunity in the TME, and present some deficiencies of CAF studies currently existed and several promising research directions in the future.

\section{Origins and activators of CAFs}

Activated by diverse signaling pathways, CAFs are derived from multiple cells of origin (FIG. 1). The presence of various cellular precursors for CAFs, which might explain why CAFs are a heterogeneous cell population, has been confirmed by a large amount of evidence $[8,30,31]$.

Tissue-resident fibroblasts, also termed quiescent fibroblasts, are one of the major sources of CAFs [32, 33]. Tissue-resident fibroblasts in distinct carcinomas are recruited and activated through the stimulation of different modulators [34], including transforming growth factor (TGF)- $\beta$ [35], hepatocyte growth factor (HGF) [36], platelet-derived growth factor (PDGF) [37], fibroblast growth factor 2 (FGF-2) [37], stromal-derived factor-1 (SDF-1) [38] and reactive oxygen species (ROS) [39, 40]. In some tumor types, stellate cells might be another source of CAFs. For instance, in liver and pancreatic cancer models, quiescent pancreatic stellate cells (PSCs) and hepatic stellate cells (HSCs) express CAF-like surface markers such as $\alpha$-SMA upon activation with TGF- $\beta$ and PDGF, which convert them into activated CAFs [41, 42]. Moreover, vitamin A deficiency has been reported to be involved in the activation of PSCs and islet stellate cells (ISCs) [43, 44]. Furthermore, a recent study revealed that the stimulation of insulin-like growth factor 1 (IGF-1) signaling is also critical for HSC activation [45]. 


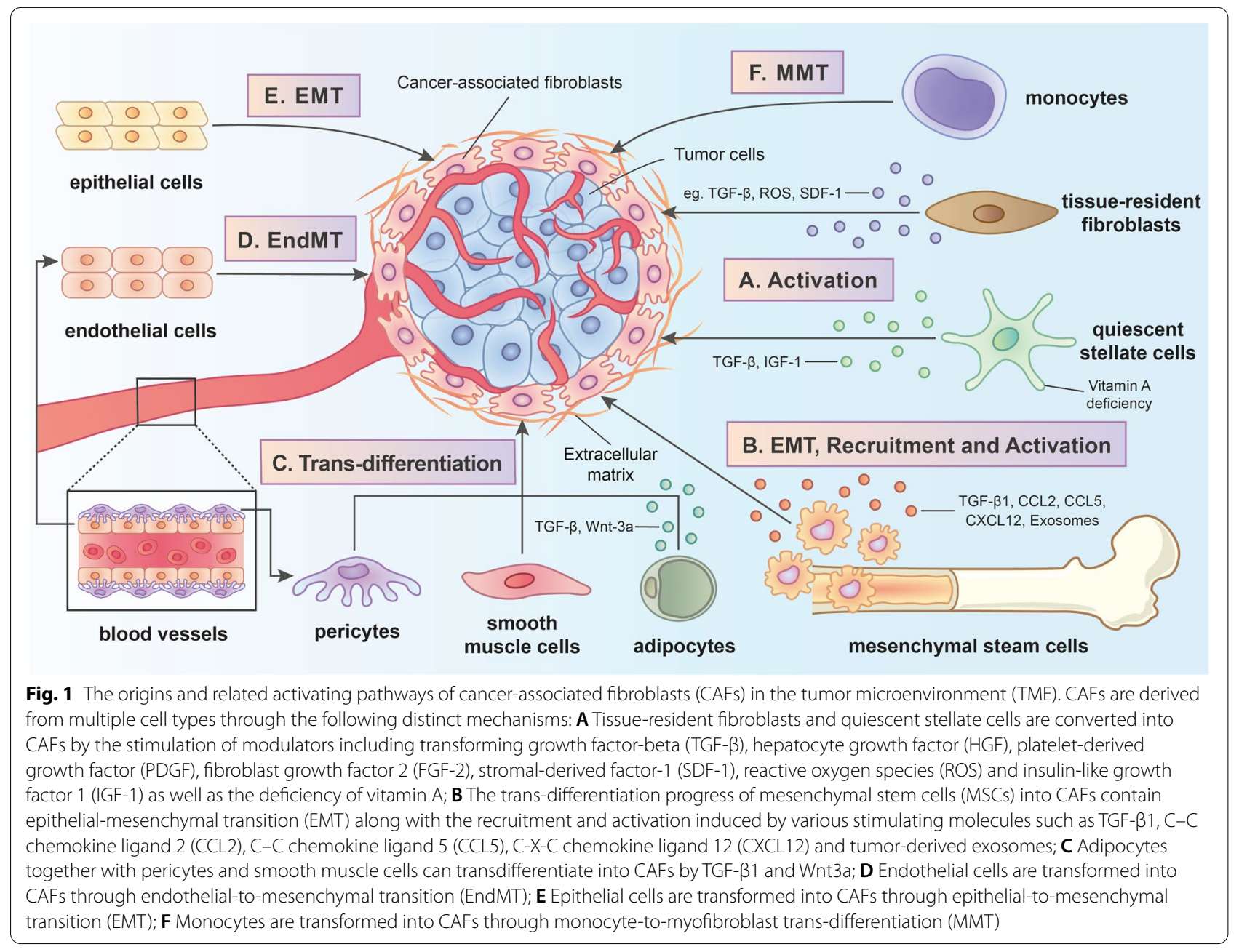

A substantial number of reports have indicated that mesenchymal stem cells (MSCs) function as precursors of CAFs $[46,47]$. The transformation of bone marrow mesenchymal stem cells (BMSCs) into CAFs might be a multistep and complicated process involving epithelialmesenchymal transition (EMT), bone marrow-derived progenitors, cell-cell communication and stimulation with various cytokines [48-50]. Effectors stimulating MSC trans-differentiation vary from cancer to cancer. In prostate carcinoma, MSCs transdifferentiate into CAFs through the activation of tumor cell- and stromal cell-secreted TGF- $\beta 1$, as well as $\mathrm{C}$-X-C chemokine ligand (CXCL) 16 [51, 52]. In breast cancer, bone marrow-derived mesenchymal stem cells (BM-MSCs) are recruited and transformed into distinct CAF subsets through the TGF- $\beta 1$-mediated osteopontin-myeloid zinc finger 1 (MZF1) pathway [53]. Subsequent studies further confirmed the importance of the TGF- $\beta$ signaling pathway in the transformation of MSCs into CAFs; for example, the pathway is involved in their metabolic reprogramming toward increased glycolysis [54]. In addition, secreted $\mathrm{C}-\mathrm{C}$ chemokine ligand (CCL) 2, CCL5 and CXCL12 in the TME are also involved in the recruitment and transformation of MSCs $[46,55]$. Notably, multiple internal mechanisms for MSC transformation might exist. For example, the differentiation of BM-MSCs into CAFs induced by cancer cells was reported to primarily depend on the Notch and Akt signaling pathways [56]. However, Peng et al. [57] discovered that tumor-derived GRP78, an endoplasmic reticulum (ER) chaperone, elicits the differentiation of BM-MSCs into CAFs in a TGF- $\beta$ / SMAD-dependent manner.

Adipocytes, especially white adipocytes [58, 59], are regarded as another cell type within CAF precursors. For instance, human adipose tissue-derived stem cells (HASCs) transdifferentiate into CAFs with a fibroblastic phenotype ( $\alpha$-SMA and tenascin-C expression) upon activation with TGF- $\beta 1$ [60]. Moreover, research in breast cancer has found that Wnt3a produced by tumor cells mediates the $\beta$-catenin-dependent differentiation 
of adipocytes into adipocyte-derived fibroblasts (ADFs), which express one CAF marker, FSP-1, at high levels $[61,62]$. Furthermore, several other potential sources of CAFs have been identified, such as epithelial cells [63, 64], pericytes [65], monocytes [66], endothelial cells [67] and smooth muscle cells [68]. These cells can be activated and differentiate into CAFs through various mechanisms. Through EMT and endothelial-to-mesenchymal transition (EndMT), most epithelial cells and endothelial cells can express a number of fibroblast markers, such as $\alpha$-SMA and FAP $[69,70]$. Peritoneal mesothelial cells, one of the special cell types among epithelial cells, are reported to be converted into CAFs through TGF$\beta 1$-induced mesothelial-mesenchymal transition (MMT) [71]. Additionally, monocytes are able to transdifferentiate into myofibroblasts through a process termed monocyte-to-myofibroblast trans-differentiation (MMT), which is induced by ROS through the p38-mitogen-activated protein kinase (MAPK) signaling pathway [66, 72].
During the generation of CAFs, various factors in the TME induce CAF activation by stimulating certain distinct signaling pathways (Table 1). In addition to the regulatory molecules described above, inflammatory mediators such as IL- $1 \beta$ and IL- 6 act through the nuclear factor-kappa B (NF- $\mathrm{kB}$ ) and Janus kinase (JAK)-signal transducer and activator of transcription 3 (STAT3) signaling pathways, respectively, to promote the malignant progression of CAFs $[29,73]$. Analogous to the classical activating mechanisms reported for normal fibroblasts, such as wound stimulation, CAFs respond to damage-associated molecular patterns (DAMPs) released by necrotic cancer cells, which subsequently induce the activation of the internal NOD-like receptor protein 3 (NLRP3) inflammasome signaling pathway and ultimately contribute to tumor growth and metastasis through the secretion of inflammasomes [74]. Furthermore, tumor-derived exosomes that contain different transmitters, such as the CD44v6/C1QBP complex, have

Table 1 Various stimulating factors and their related activating mechanisms during cancer-associated fibroblast progression

\begin{tabular}{|c|c|c|c|c|c|}
\hline Stimulating factors & Types & Activating mechanisms & Biology effects & Cancer models & Refs \\
\hline TGF- $\beta 1$ & Growth factor & $\begin{array}{l}\text { TGF- } \beta 1-S M A D \text { signaling } \\
\text { pathway }\end{array}$ & $\begin{array}{l}\text { Induces tumor cell proin- } \\
\text { vasive properties }\end{array}$ & $\begin{array}{l}\text { Colon cancer, Breast } \\
\text { cancer }\end{array}$ & {$[35,38]$} \\
\hline SDF-1 (CXCL12) & Chemokine & $\begin{array}{l}\text { SDF-1-CXCR4 autocrine } \\
\text { signaling pathway }\end{array}$ & $\begin{array}{l}\text { Maintains myofibroblast } \\
\text { activation }\end{array}$ & Breast cancer & {$[38]$} \\
\hline$I L-1 \beta$ & Proinflammatory cytokine & NF-kB signaling pathway & $\begin{array}{l}\text { Enhances tumor-promot- } \\
\text { ing inflammatory response }\end{array}$ & Squamous skin carcinoma & [29] \\
\hline IL-6 & Proinflammatory cytokine & $\begin{array}{l}\text { JAK-ROCK-STAT3 signaling } \\
\text { pathway }\end{array}$ & $\begin{array}{l}\text { Facilitates CAF-induced } \\
\text { ECM remodeling }\end{array}$ & Melanoma & [73] \\
\hline DAMPs & $\begin{array}{l}\text { Cell-necrosis-associated } \\
\text { product }\end{array}$ & $\begin{array}{l}\text { NLRP3 inflammasome } \\
\text { signaling pathway }\end{array}$ & $\begin{array}{l}\text { Promotes tumor growth } \\
\text { and metastasis }\end{array}$ & Breast cancer & [74] \\
\hline CD44v6/C1QBP complex & PDAC-derived exosomes & IGF-1 signaling pathway & $\begin{array}{l}\text { Induces HSC activation, } \\
\text { ECM remodeling and liver } \\
\text { metastasis }\end{array}$ & PDAC & [45] \\
\hline HSF-1 & Transcription factor & $\begin{array}{l}\text { Wnt and YAP/TAZ signaling } \\
\text { pathway }\end{array}$ & $\begin{array}{l}\text { Facilitates CAF aggressive } \\
\text { behaviors (mediating ECM } \\
\text { remodeling, cancer cell } \\
\text { growth and invasion) }\end{array}$ & $\begin{array}{l}\text { Breast, colorectal and ovar- } \\
\text { ian cancer }\end{array}$ & [76] \\
\hline LIF & Multifunctional cytokine & Epigenetic switch & $\begin{array}{l}\text { Initiates and maintains CAF } \\
\text { proinvasive phenotypes, } \\
\text { promotes tumor invasion } \\
\text { and ECM remodeling }\end{array}$ & $\begin{array}{l}\text { Head and neck, lung and } \\
\text { breast cancer }\end{array}$ & {$[77,78]$} \\
\hline ROS & Oxidative stress molecule & $\begin{array}{l}\text { Autophagy and caveolin-1 } \\
\text { dependent pathway }\end{array}$ & $\begin{array}{l}\text { Promotes tumor migra- } \\
\text { tion, invasion and ECM } \\
\text { remodeling }\end{array}$ & Breast cancer & {$[40,79]$} \\
\hline Matrix stiffening & Environmental stressor & SFK-YAP signaling pathway & $\begin{array}{l}\text { Establishes a feed-forward } \\
\text { self-reinforcing loop to } \\
\text { maintain CAF phenotypes } \\
\text { and enhance matrix } \\
\text { remodeling }\end{array}$ & Breast cancer & [81] \\
\hline
\end{tabular}


exhibited significant facilitation of the activation of HSCs and thus direct CAFs to induce tumor metastasis as well as ECM remodeling [45]. Moreover, heat shock factor 1 (HSF1, a master regulator of the heat shock response) was also reported to primarily orchestrate concomitant stimulation of both the $\beta$-catenin and YAP/TAZ signaling pathways through Dickkopf-3 (DKK3, an HSF1 effector), consequently resulting in aggressive behaviors of CAFs $[75,76]$. Of note, epigenetic changes are capable of initiating and maintaining a proinvasive phenotype of CAFs [77]. Albrengues et al. [77, 78] revealed that leukemia inhibitory factor (LIF) can induce a series of internal epigenetic modifications in fibroblasts, including alterations in STAT3 acetylation, phosphatase methylation of SH2containing protein tyrosine phosphatase-1 (SHP-1) and JAK1 phosphorylation, ultimately stimulating the JAK1/ STAT3 signaling pathway, which sustains the proinvasive activities of CAFs. Finally, CAF activation also depends on environmental stressors (ROS, matrix stiffening, etc.) [79-84] and DNA damage during radiation therapy [85].

Although researchers have recently employed advanced technology, such as genetic lineage tracing [86], the origins of CAFs among most cancer types remain elusive due to the lack of exclusive markers for normal fibroblasts and CAFs [87]. Lineage tracing methods combined with single-cell spatial analyses are urgently needed to identify the exact contribution of each cell type and illustrate the detailed mechanism of CAF activation during cancer development.

\section{Heterogeneity and plasticity of CAFs}

Due to the existence of multiple types of cellular precursors, the CAF population behaves as complex cells with various fibroblast phenotypes and distinct functions among many cancer types [88] (Table 2). During the past several years, several subtypes of CAFs in pancreatic ductal adenocarcinoma (PDAC) have been identified through the application of transcriptome analyses, but none of these subtypes was given a specific definition [89, 90]. Öhlund and colleagues [91] first discovered and identified two spatially divided and totally opposite subsets of CAFs-myofibroblastic CAFs (myCAFs) and inflammatory CAFs (iCAFs). myCAFs are located in direct proximity to cancer cells and have high $\alpha$-SMA expression; iCAFs are located far more distantly from neoplastic cells and express less $\alpha$-SMA but secrete more IL- 6 and other inflammatory factors (e.g., IL-8, IL-11 and LIF), and they might participate in immune suppression and tumor cachexia by stimulating the STAT3 signaling pathway. Subsequently, the presence of myCAFs and iCAFs in pancreatic cancer was confirmed through dropletbased single-cell transcriptomics technology [92]. Notably, the researchers also termed a newly discovered CAF subpopulation "antigen-presenting CAFs" (apCAFs), and these cells express MHC class II and CD74 instead of classical costimulatory molecules. Coincidentally, a subpopulation observed previously that was able to present antigens and contribute to the suppression of T cellmediated antitumor responses was analogous to apCAFs [93]. Another study in PDAC reported similar subtypes of the CAF population described above. According to the results of single-cell RNA sequencing, fibroblast population 1 (FB1) and fibroblast population 3 (FB3) [94] might represent the previously described $\mathrm{iCAF}$ and myCAF populations, respectively. Interestingly, in that study, the researchers found that FB3 also processed and presented antigens by expressing numerous MHC-II-associated genes, indicating that FB3 might be a mixed population comprising myCAFs and apCAFs. Furthermore, a recent study further assessed the intertumor and intratumor heterogeneity of human PDAC-derived CAFs [95]. At least four subtypes of CAFs were characterized by different mRNA expression profiles, and periostin (POSTN), myosin-11 (MYH11), and podoplanin (PDPN) were selected as biomarkers for subtype A to C CAFs. Moreover, subtype A CAFs, which are located in the tumor invasive front, are associated with tumor capsule formation and metastatic progression. The subtype B population might be related to a poor prognosis, while subtype C CAFs appear to be related to a favorable clinical prognosis of patients with cancer. Various CAF subpopulations have been reported in human breast cancer. For example, four different CAF subsets (S1-S4) are classified based on their diverse expression of fibroblast markers (e.g., CD29, FAP, $\alpha$-SMA, PDGFR $\beta$, FSP1 and caveolin 1 (CAV1)) [96]. Both the CAF-S1 and CAF-S4 subsets exhibit protumorigenic properties, while the CAF-S1 subset enhances the differentiation, recruitment and activation of Treg cells, thereby facilitating immune suppression of tumors; the properties of this CAF-S1 subset are similar to those of the CAF-S1 subset observed in ovarian cancer [97]. Another study on axillary lymph nodes [98] further indicated that the CAF-S1 subset promotes cancer cell migration and EMT initiation mainly by secreting CXCL12 and TGF- $\beta$, while the CAF-S4 subset facilitates the migration and invasion of tumor cells through the NOTCH pathway. Additionally, the presence of myCAFs, iCAFs and apCAFs in breast cancer was recently documented, and these cells were previously identified in PDAC [99]. In addition, Bartoschek et al. [100] defined another four subpopulations of CAFs-vCAFs, mCAFs, cCAFs and dCAFs-according to their distinct cellular sources using single-cell RNA sequencing. vCAFs, $\mathrm{mCAFs}$, and dCAFs appear to originate from perivascular cells, resident fibroblasts, and malignant cells, respectively. In addition, CAF subpopulations with different 
Table 2 Phenotypic and functional heterogeneity of cancer-associated fibroblasts exhibited in distinct tumor types

\begin{tabular}{|c|c|c|c|c|}
\hline Cancer types & Subtypes & $\begin{array}{l}\text { Characteristic markers/ expression/ } \\
\text { secretion }\end{array}$ & Functions & Refs \\
\hline \multirow[t]{3}{*}{ PDAC } & myCAFs (pCAFs) & $\begin{array}{l}\text { a-SMA, TAGLN, MYL9, TPM1, TPM2, POSTN } \\
\text { and MMP11 }\end{array}$ & $\begin{array}{l}\text { Tumor proliferation, Migration, Invasion and } \\
\text { ECM remodeling }\end{array}$ & {$[92,93,103]$} \\
\hline & iCAFs (pCAFs) & $\begin{array}{l}\text { PDGFRa, HAS1, HAS2, IL-6, IL-8, IL-11, CXCL1, } \\
\text { CXCL2 and CCL2 }\end{array}$ & $\begin{array}{l}\text { Immune suppression, Cachexia and Chem- } \\
\text { oresistance }\end{array}$ & {$[92,93,103]$} \\
\hline & apCAFs (pCAFs) & MHC class II, H2-Aa, H2-Ab1 and CD74 & Antigen-present, Immune modulation & {$[93,94,103]$} \\
\hline \multirow[t]{3}{*}{ PDAC } & CAF-A & POSTN & Tumor proliferation, Invasion, Metastasis & {$[95]$} \\
\hline & CAF-B & MYH11 & $\begin{array}{l}\text { Lymph-node metastasis, Prognostic factor } \\
\text { (adverse) }\end{array}$ & {$[95]$} \\
\hline & CAF-C & PDPN & $\begin{array}{l}\text { Immune promotion, Prognostic factor } \\
\text { (favorable) }\end{array}$ & {$[95]$} \\
\hline \multirow[t]{4}{*}{ Breast cancer } & CAF-S1 & CD29, FAP, a-SMA, PDGFRß, FSP1 and CXCL12 & $\begin{array}{l}\text { Tumor proliferation, Migration, Lymph-nodes } \\
\text { metastasis, Immune suppression and EMT } \\
\text { initiation }\end{array}$ & {$[96,98]$} \\
\hline & CAF-S2 & Not reported & Not reported & {$[96,98]$} \\
\hline & CAF-S3 & CD29, FSP1, PDGFRß & Not reported & {$[96,98]$} \\
\hline & CAF-S4 & CD29, FSP1, PDGFR $\beta$ and $a-S M A$ & $\begin{array}{l}\text { Tumor invasion, Migration, Lymph-nodes } \\
\text { metastasis }\end{array}$ & {$[96,98]$} \\
\hline \multirow[t]{3}{*}{ Breast cancer } & myCAFs & $\begin{array}{l}\text { a-SMA, ACTA2, TAGLN, MYL9, IGFBP-3 and } \\
\text { TNC }\end{array}$ & $\begin{array}{l}\text { Tumor proliferation, Migration, Invasion, } \\
\text { Angiogenesis and EMT }\end{array}$ & [99] \\
\hline & iCAFs & Ly6c1, CLEC3B, HAS1, DPT and COL14A1 & $\begin{array}{l}\text { Tumor proliferation, Metastasis, Angiogen- } \\
\text { esis, Immune evasion and Chemoresistance }\end{array}$ & {$[99]$} \\
\hline & apCAFs & $\begin{array}{l}\text { CD74, H2-Aa, H2-Ab1, H2-Eb1, KRT18 and } \\
\text { FSP1 }\end{array}$ & Antigen-present, Immune modulation & {$[99]$} \\
\hline \multirow[t]{3}{*}{ Breast cancer } & $\begin{array}{l}\text { vCAFs/cCAF (prolifera- } \\
\text { tive segment of vCAFs) }\end{array}$ & $\begin{array}{l}\text { Notch3, EPAS1, COL18A1 and NR2F2 (perivas- } \\
\text { cular cells) }\end{array}$ & Angiogenesis & {$[100]$} \\
\hline & mCAFs & $\begin{array}{l}\text { Fibulin-1, PDGFRa and CXCL14 (resident } \\
\text { fibroblasts) }\end{array}$ & Immune regulation & {$[100]$} \\
\hline & dCAFs & SCRG1 (malignant cells) & Not reported & {$[100]$} \\
\hline Breast cancer & $\mathrm{CD} 10^{+} \mathrm{GPR} 77^{+}$ & CD10 and GPR77 & Chemoresistance & {$[104]$} \\
\hline \multirow[t]{2}{*}{ OSCC } & CAF-N & HA, MMPs & Tumor invasion, Immunosuppression & {$[101]$} \\
\hline & CAF-D & TGF- $\beta$ & Tumor migration & {$[101]$} \\
\hline \multirow[t]{2}{*}{ Colorectal cancer } & CAF-A & MMP2, DCN, aFAP and COL1A2 & ECM remodeling & {$[102]$} \\
\hline & CAF-B & a-SMA, ACTA2, TAGLN and PDGFA & Not reported & {$[102]$} \\
\hline \multirow[t]{4}{*}{ HGSOC } & CAF-S1 & $\begin{array}{l}\text { CD29, FAP, aSMA, FSP1, PDGFR } \beta \text { and } \\
\text { CXCL12 } \beta\end{array}$ & Tumor proliferation, Immune suppression & {$[97]$} \\
\hline & CAF-S2 (non-activated) & Not reported & Not reported & {$[97]$} \\
\hline & CAF-S3 (non-activated) & CD29, FSP1 and PDGFR $\beta$ & Not reported & {$[97]$} \\
\hline & CAF-S4 & CD29, aSMA, FSP1 and PDGFR $\beta$ & Tumor proliferation & {$[97]$} \\
\hline $\begin{array}{l}\text { PDAC/Oral/Colon/Blad- } \\
\text { der/Intestinal cancers }\end{array}$ & rCAFs & Meflin, BMP-4, Hedgehog and IKK $\beta$ & Antitumoral effect & {$[10,105-111]$} \\
\hline
\end{tabular}

\footnotetext{
Multiple phenotype and function heterogeneous cancer-associated fibroblast subsets in distinct tumor types
}

$P D A C$ pancreatic ductal adenocarcinoma, myCAFs myofibroblastic cancer-associated fibroblasts, iCAFs inflammatory cancer-associated fibroblasts, apCAFs antigenpresenting cancer-associated fibroblasts, $P C A F$ s cancer-promoting cancer-associated fibroblasts, $a$-SMA alpha smooth muscle actin, TAGLN transgelin, MYL9 myosin light chain 9, TPM1 tropomyosin 1, TPM2 tropomyosin 2, POSTN periostin, MMP11 matrix metalloproteinase 11, PDGFRa platelet-derived growth factor receptor alpha, HAS1 hyaluronan synthase 1, HAS2 hyaluronan synthase 2, IL-6 interleukin-6, IL-8 interleukin-8, IL-11 interleukin-11, CXCL1 C-X-C chemokine ligand 1, CXCL2 C-X-C chemokine ligand 2, CCL2 C-C chemokine ligand 2, MHC class II major histocompatibility complex class II, H2-Aa histocompatibility 2 class II antigen A alpha, H2-Ab1 histocompatibility 2, class II antigen A, beta 1, CD74 cluster of differentiation 74, ECM extracellular matrix, MYH11 myosin-11, PDPN podoplanin, CD29 cluster of differentiation 29, FAP fibroblast activation protein, PDGFR $\beta$ platelet-derived growth factor receptor $\beta$, FSP1 fibroblast-specific protein 1, CXCL12 C-X-C chemokine ligand 12, EMT epithelial-mesenchymal transition, ACTA2 actin alpha 2, IGFBP-3 IGF-binding protein 3, TNC Tenascin-C, Ly6c1 lymphocyte antigen 6 complex, locus C1, CLEC3B C-type lectin domain family 3, member B, DPT dermatopontin, COL14A1 collagen type XIV alpha 1, H2-Eb1 histocompatibility 2, class II antigen E, beta 1, KRT18 keratin 18, EPAS1 endothelial PAS domain protein 1, COL18A1 collagen, type XVIII, alpha 1, NR2F2 nuclear receptor subfamily 2 group F member 2, CXCL14 C-X-C chemokine ligand 14, SCRG1 scrapie responsive gene 1, CD10 cluster of differentiation 10, GPR77 G protein-coupled receptor 77 , OSCC oral squamous cell carcinoma, HA hyaluronan, TGF- $\beta$ transforming growth factor beta, MMP2 matrix metalloproteinase 2, DCN decorin, COL1A2 collagen type 1 Alpha 2 , PDGFA platelet derived growth factor $\mathrm{A}, H G S O C$ high-grade serous ovarian cancer, $r C A F s$ cancer-restraining cancer-associated fibroblasts, $B M P-4$ bone morphogenetic protein 4 , IKK $\beta$ inhibitor kappa B kinase beta 
fibroblast phenotypes have also been detected in oral squamous cell carcinoma (OSCC) [101], colorectal cancer [102] and mesenchymal high-grade serous ovarian cancer (HGSOC) [97].

CAFs are composed of not only heterogeneous subsets with distinct phenotypes but also heterogeneous subpopulations with diverse functions $[9,30]$. Observations indicate that two functionally different populations of CAFs, cancer-promoting CAFs (pCAFs) and rCAFs, may exist [112]. Generally, most CAF subsets function as pCAFs rather than rCAFs. Studies have revealed that pCAFs mainly express FAP- $\alpha$ or $\alpha$-SMA to suppress antitumor immunity through multiple pathways $[38,93,96$, $103,113]$. Modulators secreted by pCAFs, such as TGF$\beta$, IL- 6 and CXCL12, are able to promote the proliferation and invasion of cancer cells [114]. However, a recent study indicated that one of the CAF subsets in PDAC that expresses meflin (one potential marker) exerts antitumor effects on both mouse models and human cells, and this subset was subsequently identified as rCAFs [10]. Importantly, the presence of rCAFs is not limited to the context of PDAC [105-107]. Patel et al. [108] reported a myofibroblastic CAF subpopulation that inhibited cancer cell stemness by secreting bone morphogenetic protein 4 (BMP-4) in oral carcinoma. In other tumor types, including colon [109], bladder [110] and intestinal cancers [111], tumor-suppressive roles of CAFs have also been reported, suggesting a wide distribution of rCAFs across various types of cancer. However, considering the lack of in-depth phenotypic and functional characterization of CAFs, further explorations of CAF heterogeneity in most other cancer types are currently extremely difficult.

As CAFs contain multiple heterogeneous subpopulations, researchers have recently debated whether these diverse subtypes are able to interconvert, which would indirectly confirm the plasticity of CAFs. Several studies indicate that the answer to these questions is "yes". For example, iCAFs in pancreatic cancer have been reported to be able to transform into myCAFs upon the activation of TGF- $\beta$ signaling or the inhibition of the IL-1-induced JAK/STAT signaling pathway, suggesting potential plasticity between these two cellular subtypes [99]. Furthermore, research has also discovered an intermediate state between the $\mathrm{ICAF}$ and myCAF phenotypes termed $\alpha$-SMA + p-STAT3 + cells, which might subsequently be a potential target for tumor immunotherapies [99]. Moreover, in colorectal cancer, CAF-A cells (one of the CAF subtypes) were reported to be capable of converting into CAF-B cells (another CAF subtype) during the transformation of normal fibroblasts into CAFs [102]. In addition to the research described above, few studies have recently reported CAF plasticity. Currently, in-depth research on many other reported important pathways, such as the epidermal growth factor receptor (EGFR), Wnt and Hippo signaling pathways [115], and improved recognition of the epigenetic regulation of CAF states are required to help improve our understanding of CAF plasticity [87].

\section{Interaction between CAFs and the immune microenvironment in tumors}

Based on accumulating evidence, CAFs in the TME play important roles in regulating the antitumor activities of tumor-infiltrating immune cells, including innate and adaptive immune cells, in the TIME $[7,116]$. In addition, they promote the expression of immune checkpoint molecules and ECM remodeling to indirectly influence the recruitment and activity of immune cells [116]. Through the secretion of cytokines, chemokines and other effector molecules, including TGF- $\beta$, CXCL2, collagens, MMPs and laminin, CAFs can prompt immune cells to participate in the occurrence and development of cancer, along with facilitating the degradation and remodeling of the ECM $[117,118]$. Of course, some noteworthy effects of several immune cells on CAFs have also been identified $[29,119]$. To date, many studies have shown that interactions between CAFs and immune cells as well as other immune components are capable of modulating the TIME and thus inhibiting the antitumor immune response (Fig. 2) [120, 121].

\section{Interaction between CAFs and innate immune cells in the TME \\ Interaction between CAFs and tumor-associated macrophages (TAMs)}

Macrophages that infiltrate tumors, known as tumorassociated macrophages (TAMs), are classified into two distinct subsets that are activated by different polarizing cytokines, termed M1 (lipopolysaccharide (LPS) alone or with Th1 cytokines) and M2 (Th2 cytokines) [122]. M1-type macrophages mainly behave as an antitumor role in the TIME by mediating antibody-dependent cellular cytotoxicity and producing ROS and tumor necrosis factor (TNF) [123], whereas M2-type macrophages exhibit tumor-promoting activity by contributing to the activation of tumor angiogenesis, immune suppression, invasion and metastasis of cancer cells and remodeling of the ECM [124, 125].

As a key component of the TIME, TAMs play critical roles in its modulation, especially in tumor immune suppression [126, 127]. TAMs are the most prominent immune cells in the vicinity of CAF-populated areas, suggesting tight interactions between these two cell types [128]. High expression of both CAF and TAM markers, such as $\alpha$-SMA, FAP and S100 calcium binding protein A4 (S100A4), along with CD163 and CD209, 
is related to worse clinical prognosis of patients with some tumors $[128,129]$. A substantial number of studies have shown that CAFs promote the recruitment of monocytes (macrophage precursors) and their differentiation into protumorigenic macrophage subsets (M2-type TAMs) via multiple regulatory molecules, thereby impairing responses from effector $\mathrm{T}$ cells and inducing immune suppression in the TME [130]. For example, in breast cancer, by secreting monocyte chemotactic protein-1 (MCP-1), SDF-1 and chitinase 3-like 1 (Chi3L1), CAFs are able to facilitate monocyte migration and enhance the tendency of these cells to polarize into the M2 phenotype [131, 132]. Furthermore, a similar effect of CAFs on TAMs was discovered in prostate carcinoma [133, 134]. Moreover, Mace et al. [135] documented the central role of CAF-derived macrophage colony-stimulating factor 1 (M-CSF1), IL-6, and CCL2 in monocyte recruitment and the increased M2/M1 macrophage ratio in pancreatic cancer. Other cytokines, including IL-8, IL-10, TGF- $\beta$ and CCL2 (in skin tumors), secreted by CAFs have also been demonstrated to promote the recruitment of monocytes and their transformation into M2 macrophages [136-138]. In addition to facilitating macrophage recruitment and trans-differentiation, more importantly, CAFs are capable of inducing the immunoinhibitory properties of TAMs. Utilizing flow cytometry analysis, Gordon et al. [139] observed increased expression of programmed cell death protein 1 (PD-1) on the cell surface of CAFinduced M2-type TAMs. A high level of PD-1 expression in TAMs was proven to be involved in both innate and adaptive antitumor immune response suppression by subsequent studies, including decreasing their own phagocytic potency against tumor cells and inhibiting T-cell infiltration and proliferation [140]. In contrast to their stimulatory effect on TAMs, CAFs might also inhibit some aspects of TAM activities. Estrogen receptor alpha $(E R \alpha)$, for instance, whose expression on CAFs suppresses macrophage infiltration and restricts prostate cancer invasion, is mediated by decreased CCL5 and IL-6 expression. Mechanistically, Mazur et al. [141] revealed the importance of FAP (a CAF marker) in the communication between CAFs and TAMs. The authors found that FAP participated in the interaction between CAFs and SR-A (class A scavenger receptors) + TAMs mainly by cleaving type I collagen and increasing macrophage adhesion.

Reciprocally, TAMs with the M2 phenotype regulate CAF activation and progression as well $[119,142]$. In the study by Comito et al. [133], aside from confirming the promoting effect of CAFs on TAMs, M2-type macrophages were also able to enhance EMT progression to stimulate CAF activation by secreting soluble factors such as IL-6 and SDF-1. Moreover, TAMs were recently shown to influence the trans-differentiation and activity of MSCs, one of the cellular precursors of CAFs [48]. For instance, Zhang et al. [143] observed that macrophages could facilitate MSCs to acquire CAF-like properties and a proinflammatory phenotype to remodel the inflammatory microenvironment, which potentiated the oncogenic transformation of gastric epithelial cells. Additionally, in an in vitro coculture study, TAM-like macrophages were reported to induce both the proliferation and invasion of CAF-like BM-MSCs, thereby contributing to the progression of neuroblastoma [48]. Subsequently, activated CAFs induced by macrophages further enhance TAM activity, and consequently make up a positive loop that promotes cancer development and immune inhibition in the TME.

Recently, studies regarding the effect of CAFs on TAMs have been continuously reported, whereas the effect of macrophages on CAFs has not been comprehensively

\footnotetext{
(See figure on next page.)

Fig. 2 Crosstalk between cancer-associated fibroblasts (CAFs) and immune components in the tumor immune microenvironment (TIME). CAFs can orchestrate an immunosuppressive TME via interacting with the immune microenvironment in tumor. Through the secretion of multiple chemokines, cytokines and other effector molecules such as transforming growth factor-beta (TGF- $\beta$ ), interleukin-6 (IL-6), C-X-C chemokine ligand 12 (CXCL12), C-C chemokine ligand 2 (CCL2), stromal-derived factor-1 (SDF-1), vascular endothelial growth factor (VEGF) along with indoleamine 2,3-dioxygenase (IDO) and prostaglandin E2 (PGE2), CAFs modulate immune cells-mediated antitumor immunity through the following pathways: Promoting the trans-differentiation or polarization of immune cells such as tumor-associated macrophages (TAMs), tumor-associated neutrophils (TANs), mast cells (MCs), dendritic cells (DCs) and T lymphocytes into certain protumorigenic cell subsets; Facilitating the activities of immune inhibitory cells in terms of recruitment, activation and immunosuppressive effects including M2-type TAMs, N2-type TANs, regulatory DCs (rDCs), regulatory T(Treg) cells and myeloid-derived suppressor cells (MDSCs); Restricting the cytotoxic activity and cytokines production of effector immune cells like natural killer (NK) cells and cytotoxic T lymphocytes (CTLs). Notably, several infiltrating immune cells such as TAMs, TANs, MCs and DCs can in turn exert promoting effect on CAFs activation and function, thereby contributing to the formation of immune suppressive loops. Moreover, CAFs can also upregulate the expression of immune checkpoint molecules such as programmed cell death protein 1 (PD-1)/ programmed death receptor ligand-1 (PD-L1) and cytotoxic T lymphocyte associate protein-4 (CTLA4)/B7 in both themselves and other cells in the TME to induce T-cells dysfunction. Meanwhile, CAFs are able to remodel extracellular matrix (ECM) to facilitate immune suppression through the production of fibronectin, collagen and metalloproteinases (MMPs) as well as the activation focal adhesion kinase (FAK) signaling pathway. Finally, immune checkpoint molecule overexpression on CAF surface as well as matrix deposition around would inhibit CAF apoptosis and facilitate their activation and function
} 


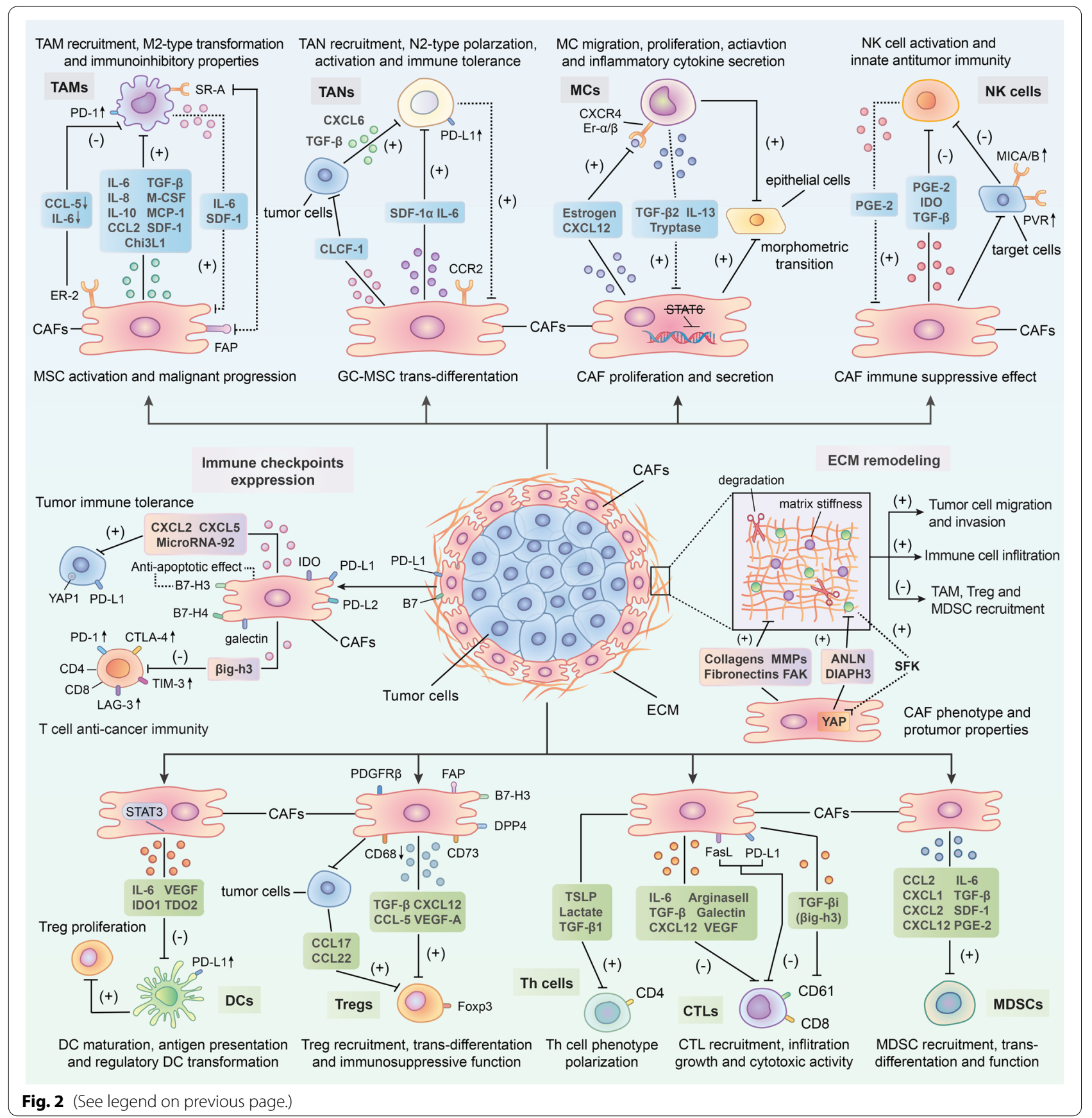

investigated and clarified. Further investigations of the mechanisms underlying CAF-TAM interactions in the TME are needed to advance current cancer-targeted therapies.

\section{Interaction between CAFs and tumor-associated neutrophils (TANs)}

Increasing evidence indicates that tumor-associated neutrophils (TANs), a significant component of the
TIME, also exhibit phenotypic heterogeneity and functional versatility $[144,145]$. Analogous to the dichotomy of TAMs (M1 and M2), neutrophils can acquire an antitumor phenotype (N1) or a protumorigenic phenotype (N2) based on whether they are activated by TGF- $\beta$ [146-148]. But unlike TAMs, the difference between N1 and N2 TAN phenotypes relies on the distinct degree of activation rather than different polarizing molecules [149]. 
Notably, CAFs might be able to modulate the polarization of TANs. As a recent study of hepatocellular carcinoma reported, CAF-derived cardiotrophin-like cytokine factor 1 (CLCF1) induces the polarization of N2-phenotype neutrophils by upregulating CXCL6 and TGF- $\beta$ expression in tumor cells, thereby facilitating tumor progression [150]. More importantly, CAFs probably participate in all stages of the malignant progression of TANs and ultimately suppress the antitumor immune response in the TME. Through the secretion of SDF-1 $\alpha$, CAFs are able to recruit peripheral neutrophils to tumors [151]. Moreover, C-X-C chemokine receptor 2 (CXCR2), a cytokine receptor that is expressed by CAFs, was proven to be a primary factor participating in the recruitment of neutrophils in tumors, indicating that CAFs might enhance the migration of TANs in a CXCR2-dependent manner [152, 153]. Next, CAF-derived IL-6 stimulates the STAT3 signaling pathway in TANs, consequently inhibiting the activity of $\mathrm{T}$ cells and inducing immune tolerance through the expression of $\mathrm{PD}-1 /$ programmed death ligand 1 (PD-L1) [151]. In addition, Zhu et al. [154] discovered a bidirectional interaction between gastric cancer mesenchymal stem cells (GC-MSCs) and neutrophils. On the one hand, GC-MSCs can induce the chemotaxis and activation of neutrophils and sustain their survival through the IL-6-mediated STAT3-extracellular regulated protein kinases $1 / 2$ (ERK1/2) axis. On the other hand, activated TANs, in turn, are capable of promoting the differentiation of MSCs into CAFs. Overall, the specific mechanisms underlying the mutual effects of CAFs and TANs on each other remain unclear due to the limited number of reports.

\section{Interaction between CAFs and mast cells (MCs)}

In recent decades, studies of mast cells (MCs) have placed more focus on their roles in cancer than on their roles in allergic diseases $[155,156]$. As a component of the TIME, interestingly, MCs exert dual effects on tumor progression-both promotion and inhibition of tumor growth-which depend on the specific MC localization, cancer type and the degree of tumor progression [157-161]. As cancer promoters, on the one hand, MCs contribute to the stimulation of angiogenesis and lymphangiogenesis along with the degradation of ECM by producing different pro-angiogenic molecules (vascular endothelial growth factor (VEGF)-A, VEGF-B, FGF-2, heparin, histamine and stem cell factor (SCF)) [162-166], lymphangiogenic molecules (VEGF-C and VEGF-D) [167], matrix metalloproteinase-9 (MMP-9) and proteases (tryptase and chymase) [168, 169]. On the other hand, as antitumor effectors, MCs produce mediators (e.g., tryptase, chondroitin sulfate, TNF, IL-1 and IL-6) that increase antitumor inflammatory reactions, inducing tumor apoptosis and decreasing the invasiveness of cancer cells $[170,171]$.

Excess numbers of CAFs and MCs in tumor islets are strongly associated with the aggressiveness of cancer, and their interactions directly contribute to tumor progression $[172,173]$. In prostate cancer, with the overexpression of estrogen inside, CAFs can potentiate MC proliferation, migration and inflammatory cytokine secretion and thus exhibit protumorigenic effects [174]. Meanwhile, CAF-derived CXCL12, induced by estrogen, was observed to be involved in the recruitment of MCs by combining with CXCR4 [174]. Furthermore, Ma et al. [175] discovered that PSCs could facilitate the activation and proliferation of MCs as well. This study also identified the stimulatory effect of MCs on CAFs. IL-13 and tryptase, which are released by $\mathrm{MCs}$, conversely stimulate CAF proliferation in a TGF- $\beta 2$-STAT6-independent manner [175]. Increasing CAFs subsequently resulted in the formation of a fibrotic TME and ultimately suppressed antitumor immunity and therapeutic responses [175]. Moreover, MCs in neurofibroma have also been reported to be capable of promoting CAF activity, such as enhancing the proliferation and secretion of CAFs through the TGF- $\beta$ signaling pathway, thereby increasing the protumor effects of CAFs [173]. Additionally, a recent study in a microtissue model of prostate cancer revealed cooperation between MCs and CAFs, which induced the early malignant morphological transition of benign epithelial cells [176]. To date, research on the correlation between MCs and CAFs in tumors is still lacking. Considering the unique role of MCs and their mediators in the TIME, studies elucidating how CAF-MC interactions are implicated in tumor immunity are required to provide better immunotherapy and clinical services.

\section{Interaction between CAFs and natural killer (NK) cells}

Natural killer (NK) cells, members of the innate immune system, naturally respond to tumor cells [177-180]. The activity of NK cells depends on the expression and stimulation of activating or inhibitory receptors on the cell surface [181]. NK cell-activating receptors include NK group 2D (NKG2D), NKp30, NKp44, NKp46 and DNAX accessory molecule 1 (DNAM-1), while killer immunoglobulin-like receptors (KIRs) and CD94/NK group 2A (NKG2A) expressed on NK cells are inhibitory receptors $[179,182]$. In solid tumors, various soluble inhibitory factors and cell types, such as CAFs, comprise the immunosuppressive TME, contributing to the impaired functionality of infiltrating NK cells $[183,184]$.

An increasing number of studies indicate that CAFs exert inhibitory effects on NK cells through multiple processes, including $\mathrm{NK}$ receptor activation, cytotoxic activity and cytokine production, in a direct or indirect 
manner [9, 185]. For example, under the influence of melanoma-associated fibroblasts, both the expression of NKp30, NKp44 and DNAM-1 activating receptors on the cell surface and the formation of cytolytic granules in NK cells are suppressed, which mainly depends on the prostaglandin E2 (PGE2) released by CAFs [186]. In hepatocellular carcinoma, CAFs educate NK cells to transition into an inactivated phenotype through PGE2 and indoleamine 2,3-dioxygenase (IDO) and create an unresponsive state in antitumor immunity [187]. Interestingly, NK cells themselves can facilitate the formation of the suppressive loop induced by CAFs via promoting the secretion of PGE2 [188]. Certainly, CAFs can also restrict the activity and function of NK cells indirectly by modulating the expression of their activating receptorassociated ligands on tumor cells. For instance, according to Ziani et al. [189], CAFs in melanoma reduce the expression of MICA/B (two ligands of NK-activating receptors) on tumor cells, thereby suppressing NKG2Ddependent cytotoxic activity and IFN- $\gamma$ secretion. Another study reported that a reduction in poliovirus receptor (PVR, a ligand of an NK-activating receptor) expression on the cell surface plays a critical role in the CAF-mediated suppression of NK cell killing activities [190]. In addition, macrophages induced by CAFs are reported to inhibit NK cell cytotoxicity and activation, which indicates that CAFs can regulate NK cells through other immune cells [137]. When cocultured with NK cells, higher PGE2 expression is detected on CAFs than on normal fibroblasts [188], suggesting that NK cells can influence the certain protein expression in CAFs as well. However, currently, only few studies have assessed the effect of NK cells on CAFs, and further investigations are needed to clarify this interacting progress.

The detailed mechanism of crosstalk between CAFs and NK cells is complicated and multiple effector molecules might participate in the interaction. TGF- $\beta$ has been widely reported to be a key cytokine connecting CAFs with NK cells in tumors [191]. Substantial studies have proven that CAF-secreted TGF- $\beta$ significantly inhibits the activation and cytotoxic activity of NK cells [192]. One of the possible mechanisms is that TGF- $\beta$ reduces the production of interferon- $\gamma$ (IFN- $\gamma$ ) and downregulates cell surface activating receptors, such as NKG2D [193, 194]. For instance, TGF- $\beta$ can inhibit DNAX-activation protein 12 (DAP12) transcription and reduce the expression of NKp30 and NKG2D by stimulating miR-183, thus silencing NK cells [195]. Moreover, Viel et al. [196] reported that TGF- $\beta 1$ selectively downregulated NKp30, NKp46, NKG2D and DNAM-1 expression in vitro through the activation of the SMAD2/3-dependent signaling pathway. In addition to TGF- $\beta$, the exploration of other related molecules is still ongoing.

\section{Interaction between CAFs and dendritic cells (DCs)}

Tumor-infiltrating dendritic cells (DCs), a heterogeneous group consisting of diverse subpopulations, play a crucial role in the activation and regulation of innate and adaptive immune responses in the TIME through the high expression of class I and class II MHC complexes, adhesion molecules and costimulatory molecules [197, 198]. In recent years, several investigations have illustrated that CAFs can drive immune evasion of tumor cells by blocking DC maturation, antigen presentation and their associated adaptive immune responses. However, their in-depth mechanisms remain unclear. By activating the IL-6-mediated STAT3 pathway, CAFs in hepatocellular carcinoma can recruit normal DCs and induce them to transdifferentiate into regulatory DCs (rDCs), disabled DCs that express costimulatory molecules at a low level and hardly present antigens, but secrete inhibitory cytokines such as IDO [199]. Further studies have revealed the importance of regulatory DC-derived IDO in the promotion of $\mathrm{T}$ cell anergy and Treg cell proliferation, which consequently results in the restriction of T cell-mediated immunity [200]. Another study of lung cancer indicated that both CAF-released IDO1 and tryptophan 2,3-dioxygenase (TDO2) induced by lung cancer-derived galectin-1 are responsible for the impaired differentiation and function of DCs through the degradation of tryptophan [201, 202]. In addition, studies have demonstrated that VEGF produced by CAFs is involved in the abnormal differentiation and impaired antigenpresenting function of DCs via inhibiting the activation of NF-kB [203, 204]. Meanwhile, VEGF is also able to facilitate immune tolerance by upregulating PD-L1 expression on the DC surface [205].

\section{Interaction between CAFs and adaptive immune cells in the TME \\ Interaction between CAFs and T lymphocytes}

$\mathrm{T}$ lymphocytes play a key role in modulating the adaptive immune response, and they comprise different subpopulations, such as Treg cells, helper $\mathrm{T}$ (Th) cells and cytotoxic T lymphocytes (CTLs) [206]. Numerous studies have illustrated the role of CAFs in modulating $\mathrm{T}$ cell activities and functions.

Treg cells with high Foxp3 expression are known to have crucial functions in the restriction of antitumor immunity [207]. Utilizing histochemical staining, Kinoshita et al. [27] confirmed that Treg cells are located adjacent to CAFs. Furthermore, the infiltration of both Foxp3+ Tregs and CAFs in the tumor stroma was correlated with a poor prognosis according to clinical data [27]. These results all indicate that potential crosstalk between CAFs and Treg cells might exist. Evidence of the interaction between CD70+CAFs and naturally 
occurring Tregs has already been reported [208]. In a study of colorectal cancer, researchers revealed that CAFs stimulate the migratory activity of Treg cells and markedly increase their frequency in tumor sites [208]. Moreover, the recruitment of CD4+CD25+ Treg cells to CAFs also depends on the chemokine CCL5 according to studies examining breast cancer [209, 210]. Other molecules, such as VEGF-A, one of the growth factors released by CAFs, have been observed to directly or indirectly participate in Treg cell induction and maintenance $[211,212]$. In addition to promoting the recruitment and infiltration of Treg cells, CAFs also promote their transformation to ultimately induce immune suppression. As shown in the study by Chen et al. [213], CAF-derived TGF- $\beta$ can facilitate the differentiation of naïve T cells into CD4 + CD25 + Treg cells by inducing the expression of the Foxp3 gene in T lymphocytes. Additionally, FAP + PDGFR $\beta+$ CAFs in breast cancer, also termed CAF-S1 cells (introduced earlier in the review), were proven to not only enhance the migration of CD4 + CD25 $+\mathrm{T}$ cells by releasing CXCL12 but also express CD73, dipeptidyl peptidase IV (DPP4) and $\mathrm{B} 7 \mathrm{H} 3$, enabling them to transform $\mathrm{CD} 4+\mathrm{T}$ cells into Foxp3 + Treg cells [96]. Recently, Zhao X and colleagues [214] discovered that downregulation of CD68 in CAFs facilitates the secretion of CCL17 and CCL22 from tumor cells and further indirectly increases the infiltration of Treg cells. However, interestingly, Özdemir et al. [105] obtained the opposite result from the experiment: the exhaustion of myofibroblasts in PDAC increases the proliferation of CD4+ Foxp3 + Tregs and subsequently inhibits immune surveillance, suggesting that a possible mixed and dual relationship might exist between CAFs and Treg cells.

Th cell subsets mainly include Th1, Th2, and Th17 cells, which are mostly differentiated from naïve CD $4+\mathrm{T}$ cells [215]. By secreting various specific cytokines, Th1 and Th2 cells participate in cellular and humoral immunity, respectively [216]. Several reports have shown the great influence of CAF-associated activities on Th cell polarization, while their specific effects remain unclear. For example, when CAF activation proteins are targeted by a DNA vaccine, the polarization of the Th2 subset is suppressed at the same time, indicating that activated CAFs might promote the differentiation above [217]. Subsequently, De Monte et al. [218] found that thymic stromal lymphopoietin (TSLP) produced by activated CAFs in pancreatic cancer functions to promote Th2 polarization. In prostate cancer, in contrast, CAFs drive the polarization of naive $\mathrm{CD} 4+\mathrm{T}$ cells from the $\mathrm{Th} 2$ to $\mathrm{Th} 1$ phenotype by stimulating the miR21/Toll-like receptor 8 (TLR8) axis through the release of lactate [219]. In addition, by producing TGF- $\beta 1$, CAFs can facilitate Th17 cell differentiation in vivo and disease development [220]. Altogether, CAFs modulate the transformation of most Th cells into immunoinhibitory subpopulations in tumors to create an immunosuppressive and cancer-adaptive TME and then exert a proinvasive effect on cancer cells.

$\mathrm{CD} 8+\mathrm{T}$ cells, also called CTLs, mediate cytotoxic activities mainly by inducing the apoptosis of tumor cells, which is considered the most critical component of antitumor immunity [221, 222]. A substantial number of studies have reported the interactions between CAFs and CD8 $+\mathrm{T}$ cells and documented the inhibitory effect of CAFs on CD8 + T cell infiltration, growth and antitumor immunity [223]. Multiple factors account for the decreased infiltration of CD8 $+\mathrm{T}$ cells in the TME. For instance, by secreting cytokines such as CXCL12, activated PSCs are able to facilitate the trafficking of CD8 $+\mathrm{T}$ cells away from the juxta-tumoral compartment and thus reduce the frequency of infiltrating CTLs in tumor islets [25]. Subsequently, the importance of the CXCL12 signaling pathway in the regulation of tumor-infiltrating $\mathrm{CD} 8+\mathrm{T}$ cell migration induced by FAP $+\mathrm{CAFs}$, has been confirmed in several reports [224, 225]. Certainly, the physical barriers and hypoxia in the TME caused by CAF-mediated ECM modification are also responsible for $\mathrm{T}$ cell movement restriction [226]. CAFs release various angiogenic factors in response to hypoxia, such as VEGF, which leads to decreased cell adhesion molecule (e.g., intercellular cell adhesion molecule (ICAM)-1/2 and vascular cell adhesion molecule-1 (VCAM-1)) expression on endothelial cells [227]. Due to the lack of cell adhesion molecules, the extravasating progress of peripheral $\mathrm{CD} 8+\mathrm{T}$ cells into tumor sites through the vasculature is hard to maintain [228]. In addition, CAFs can also reduce $\mathrm{CD} 8+\mathrm{T}$ cell recruitment by releasing IL- 6 and TGF- $\beta$, and inhibit their cytotoxic activities toward tumor cells as well $[113,229]$. Further related clinical trials have indicated that IL- 6 blockade therapy effectively improves the function of $\mathrm{T}$ cells and the prognosis of patients with cancer [113, 229]. According to the research of Goehrig et al. [230], CAFs can exert a direct suppressive effect on $\mathrm{CD} 8+\mathrm{T}$ cell function, including their proliferation, activation and cytotoxic activity, through the secretion of $\beta i g-h 3$ (one ECM protein, also termed TGF- $\beta \mathrm{i}$ ). Mechanistically, CAF-derived $\beta$ ig-h3 induces the combination of hydrogen peroxide inducible clone-5 (HIC-5) protein and Y505 phosphorylated Lck by binding to CD61 (one $\mathrm{CD} 8+\mathrm{T}$ cell surface marker) and consequently decreases the transduction of $\mathrm{T}$ cell receptor (TCR) signaling [230]. Moreover, arginase II and galectin expressed in CAFs are also involved in the progression of suppressing CD8 $+\mathrm{T}$ cell proliferation and promoting T cell anergy [231-233]. Of note, as previously described, CAFs are capable of inhibiting $\mathrm{CD} 8+\mathrm{T}$ cell cytotoxic function in indirect 
manners. CAFs not only blunt antigen presentation of DCs or NK cells by disturbing their normal differentiation [187, 199], but also induce immunoinhibitory subsets (e.g., TAMs and Treg cells) and immune checkpoint expression to impair effector $\mathrm{T}$ cell antitumor responses [130, 151]. Recently, in-depth research has revealed a possible suppressive mechanism by which CAFs in the TME might function in a similar manner to normal DCs, including participating in antigen sampling, processing and presentation and upregulating the expression of immune checkpoint molecules (factor associated suicide (FAS)/factor associated suicide ligand (FASL) and PD-1/ programmed death ligand 2 (PD-L2)), thereby promoting a decrease in the number of $\mathrm{CD} 8+\mathrm{T}$ cells and an increase in tumor cell viability [93]. Since CAFs can suppress the immune reaction in the TME by regulating the properties of various $\mathrm{T}$ cell subsets, targeted immunotherapies aimed at the CAF-T cell interaction might be effective at stimulating an impaired antitumor response.

In conclusion, CAFs facilitate the cancer-promoting phenotype transition of naïve $\mathrm{T}$ cells, enhancing immune inhibitory $\mathrm{T}$ lymphocyte function and suppressing the activity of effector $\mathrm{T}$ lymphocytes, thereby resulting in immune suppression in the TME. Currently, there is still a lack of studies reporting the effect of $\mathrm{T}$ lymphocytes on CAFs, which might be a novel potential direction for future research.

\section{Interaction between CAFs and MDSCs}

Originating from bone marrow, MDSCs are famous for their strong immunosuppressive activity in the TIME [234]. MDSCs mainly contain two cell subsets, termed polymorphonuclear MDSCs (PMN-MDSCs) and monocytic MDSCs (M-MDSCs), which are phenotypically and morphologically similar to neutrophils and monocytes, respectively [235, 236]. In contrast to MDSCs that are activated by bacteria and viruses, MDSCs in the TME exhibit less phagocytic activity while continuously releasing anti-inflammatory cytokines, ROS and nitric oxide (NO), thereby contributing to the promotion of cancer angiogenesis, invasion, metastasis and immune tolerance [237-239].

Recently, a novel MDSC subset, named circulating fibrocytes, was reported to exhibit phenotypic and functional similarity to CAFs, suggesting a possible association between MDSCs and CAFs [240]. By releasing various cytokines and chemokines, CAFs can facilitate the infiltration and generation of MDSCs and consequently suppress effector $\mathrm{T}$ cell antitumor activity. Evidence indicates the essential role of CCL2 in the recruitment of both PMN-MDSCs and M-MDSCs [28, 241]. As a major source of CCL2, CAFs might induce MDSCs to migrate into tumor sites by stimulating the
STAT3 signaling pathway [225]. For example, CAFs in lung squamous cell carcinoma (LSCC) have been reported to promote peripheral $\mathrm{C}-\mathrm{C}$ chemokine receptor (CCR) $2+$ monocyte migration via CCL2 and then reprogram them into M-MDSCs [242]. The accumulation of immunoinhibitory subpopulations (M-MDSCs) in the TME finally contributes to $\mathrm{CD} 8+\mathrm{T}$ cell growth and IFN- $\gamma$ production restriction [242]. Moreover, in hepatic carcinoma, Deng et al. [243] found that recruited monocytes can differentiate into M-MDSCs, and this transformation is induced by CAFs through IL-6 in a STAT3-dependent manner, which subsequently results in extensive inhibition of $\mathrm{T}$ cell proliferation and function. Another study described similar effects of CAFsecreted CXCL12 on monocytes in triple-negative (TN) breast cancers [244]. Recent research in esophageal squamous cell carcinoma confirmed the importance of CAFsecreted IL-6 in MDSC differentiation and observed that CAF-derived exosome-packed microRNA-21 (miR-21) is also responsible for the generation of M-MDSCs via activating STAT3 signaling [245]. In addition, under the inhibitory action of tranilast (a CAF suppressor), the expression of CAF-derived SDF-1, PGE2 and TGF- $\beta 1$ is decreased, along with a low-level differentiation of original MDSCs [246]. These findings indicate that SDF-1, PGE2 and TGF- $\beta 1$ probably participate in the differentiation and modulation of MDSCs [246]. Finally, CXCL1, a granulocytic chemokine produced by CAFs, might also be involved in PMN-MDSC recruitment [247].

\section{Interaction between CAFs and other immune cells}

Certainly, other immune cells, such as monocytes and B cells, can crosstalk with CAFs as well. As we described above, CAFs are able to facilitate monocyte migration and trans-differentiation into M2-type TAMs [131, 132]. For B cells, only CXCL13 secreted by CAFs has been reported to enhance the recruitment of $B$ cells [116]. Moreover, no other study has reported CAF-B cell interactions.

\section{Interaction between CAFs and other immune components in the TME}

\section{CAFs upregulate the expression of immune checkpoint} molecules on the cell surface to induce immunologic tolerance

High expression of immune checkpoint molecules on the surface of T-cells and tumor cells has been identified as a main contributor to the dysfunction of $\mathrm{T}$ lymphocytes in the TME [248-251]. PD-L1 and PD-1, for example, are well-known checkpoint molecules. The binding of PD-L1 to its receptor PD-1 on activated T cells hampers antitumor immunity by counteracting $\mathrm{T}$ cell-activating signals [252]. 
CAFs themselves can express different ligands of immune checkpoint molecules on their cell surface, including PD-L1, PD-L2, B7-H3/H4, galectins and the enzyme IDO [93, 253-256]. Studies have demonstrated that the overexpression of PD-L1 and PD-L2 on CAFs among colon tumors, melanoma, carcinomas and lung cancer substantially induces $\mathrm{T}$ cell exhaustion and deactivation [93, 257-259]. Furthermore, $\alpha$-SMA + CAFs expressing high levels of $\mathrm{B} 7-\mathrm{H} 3$ were recently shown to exhibit prolonged survival because of the antiapoptotic effect of this checkpoint molecule, and its presence also predicts a poor prognosis of gastric adenocarcinomas (GACs) $[255,260]$.

In addition to the upregulation of molecules on their own surface, CAFs also produce various types of cytokines and exosomes to upregulate checkpoint molecules on other cells, such as tumor cells and immune cells in the TME, which indirectly exert inhibitory effects on $\mathrm{T}$ cell function and antitumor responses. For instance, CAFs in pancreatic cancer have been reported to upregulate the expression of certain immune checkpoint molecules, including PD-1, cytotoxic lymphocyte-associated antigen-4 (CTLA-4), T cell immunoglobulin, mucindomain containing-3 (TIM-3) and lymphocyte-activation gene-3 (LAG-3), on both CD4+and CD8 + T cell surfaces, which consequently inhibits proliferating $\mathrm{T}$ cells and their specific recognition of tumor cells [261]. During the regulation of immune checkpoints, CAF-derived Bigh3 might play a crucial role in promoting the expression of certain immune checkpoint molecules [230]. When applying $\beta$ ig-h3-targeted depleting Ab therapy, researchers observed the reduced expression of PD-1 and TIM-3 on the tumor-specific CD8 + T cell surface along with the recovery of their proliferation and activity [230]. Moreover, IL-6 secreted by CAFs, as described before, can induce PD-L1 expression on neutrophils by activating the STAT3 signaling pathway to restrict effector $\mathrm{T}$ cell function [151]. Interestingly, CAF-derived factors involved in the upregulation of PD-L1 in different tumor cell types are distinct. Through the secretion of soluble factors like CXCL2, $\alpha$-SMA + CAFs can increase PD-L1 expression in lung adenocarcinoma cells, thereby influencing antitumor immunity [262]. In melanoma and colorectal carcinoma, Li et al. [263] found that CAF-derived CXCL5 was involved in the expression of PD-L1 on the tumor cell surface in a PI3K/AKT-dependent manner. Recent studies have revealed some detailed intracellular signaling mechanisms. As shown in the research by Zhang et al. [264], CAFs in colorectal cancer facilitate extracellular signal regulated kinase 5 (ERK5) expression and phosphorylation to increase the synthesis of PD-L1 protein. Additionally, in human breast cancer, studies recently confirmed that microRNA-92 in CAF-derived exosomes targets LATS2 (a target gene of miR-92) and enhances the nuclear translocation of yes-associated protein 1 (YAP1); in this way, YAP1 binds to the enhancer region of PD-L1 to promote its transcriptional activity [265]. However, less is currently known about CAF induction of immune checkpoint molecule expression on other cells in the TME.

Overall, CAFs not only induce endogenous overexpression of checkpoint molecule ligands but also upregulate the expression of immune checkpoint molecules on other cells in the TME, thereby contributing to the impaired function of tumor-infiltrating $\mathrm{T}$ lymphocytes and immunologic tolerance. Certainly, further studies are needed to clarify the deeper mechanisms of CAF-induced immune checkpoint molecule expression, which might be a potential target for CAF-specific immunotherapies.

\section{CAFs remodel the extracellular matrix (ECM) to facilitate immune suppression}

The extracellular matrix (ECM) is a complex network consisting of different macromolecules, including collagens, fibrin, glycoproteins and proteoglycans, responsible for maintaining the architecture, integrity, development and homeostasis of normal tissue [18, 266, 267]. ECM alteration in the TME is a common phenomenon in tumor tissues and is usually related to cancer progression [268]. Many studies have demonstrated the pivotal role of CAFs in remodeling the $\operatorname{ECM}[17,269]$. By secreting multiple matrix proteins (e.g., fibronectin and type I collagen) and producing a variety of matrix metalloproteinases (MMPs), such as MMP-1 and MMP3, CAFs can facilitate the degradation of normal ECM structure along with increasing matrix stiffness [270274]. Moreover, CAFs also release the cytokine TGF- $\beta 1$, a growth factor that is reported to be one of the most important regulators during ECM remodeling [275, 276]. The modified ECM, in turn, exerts promoting effects on CAF activation and protumorigenic function. A positive feedback loop between CAFs and the ECM has been identified by Calvo et al. [81]. Through activated YAP, CAFs are capable of upregulating the expression of several cytoskeletal regulators (e.g., anillin (ANLN) and diaphanous-related formin-3 (DIAPH3)) to contribute to ECM stiffening [81]. When the matrix becomes stiffer in the ECM, isometric tension within CAFs significantly increases and further facilitates YAP activation by stimulating Src family kinases (SFKs), consequently maintaining the CAF phenotype and their cancer-promoting properties [81].

Based on accumulating evidence, the modified ECM induced by CAFs is associated with the migration and invasion of cancer cells [17, 277, 278]. More importantly, this modified matrix participates in the induction 
of immune suppression within the TME. The CAFremodeled ECM protein network serves as a physical barrier for immune cells, especially $\mathrm{T}$ lymphocytes, thus inhibiting their recruitment into cancer sites and subsequently reducing their opportunities to participate in the immune response in the TME $[279,280]$. The collagen density of the ECM is able to determine the $\mathrm{T}$ cell distribution in the TME. Increased collagen deposition surrounding tumor cell clusters in lung tumors and pancreatic cancers was observed to restrict $\mathrm{T}$ lymphocyte access to contacting cancer cells $[281,282]$. In addition, the accumulation of numerous matrix proteins in the ECM also results in a chronic hypoxia state in the

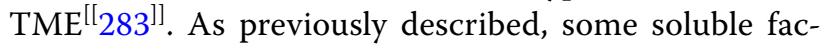
tors such as VEGF induced by hypoxia can decrease the effusion rate of circulatory $\mathrm{T}$ cells through tumor vessels and then reduce their infiltration [227, 228]. Further study revealed the critical role of focal adhesion kinases (FAKs, nonreceptor tyrosine kinases, including FAK1 and PYK2/FAK2), as fibrotic regulators, in the poor infiltration of CD8 + cytotoxic $\mathrm{T}$ cells induced by CAFdirected matrix deregulation [284]. The fibrous stroma of the ECM around tumor islets often blocks highmolecular-weight drugs and thus decreases the efficacy of cancer chemotherapy [285]. Recent studies have indicated that FAK-targeted inhibition can decrease the stromal density and consequently increase the responsiveness of tumors to chemotherapy and immunotherapy, suggesting that it might be a potential therapeutic target for tumor chemoresistance [286].

In addition, the CAF-modified ECM can modulate the activities of other immune cell populations as well. Abnormal cancerogenic collagenous matrix is involved in TAM recruitment and function [287]. For instance, the collagen-rich matrix induced by CAFs not only promotes monocyte migration and proliferation, but also shifts macrophage differentiation to M2 polarization (a protumorigenic cell subset) [288-291]. Reciprocally, TAM direct or indirect modulatory regulation of collagen deposition and geometrical organization gradually increase matrix rigidity and ultimately accelerate ECM remodeling progress [287]. Moreover, the ECM also facilitates the infiltration of other immunoinhibitory subpopulations. Increased collagen density or stiffness in the ECM leads to extensive FAK activation within cells, and activated FAKs subsequently drive the direct exhaustion of CD8 $+\mathrm{T}$ cells and enhance the recruitment of Tregs, MDSCs and TAMs, thereby contributing to the formation of an immunosuppressive TME [292, 293]. Altogether, the ECM has been demonstrated to crosstalk with several immune cells to induce immune suppression, whereas the effect of the ECM on other cell types, such as DCs and TANs, remains unclear.

\section{Therapeutic strategies for targeting CAFs to enhance the anticancer immune response}

With in-depth research and an understanding of the immune response suppression driven by CAFs, these cells are becoming one of the most promising therapeutic targets for cancer intervention. In recent decades, the number of preclinical experiments that restore the anticancer immune response through CAF-targeted therapies has increased dramatically. Currently, there are three main strategies for CAF-based immunotherapy: direct CAF depletion, CAF activation and functional suppression along with CAF-induced ECM remodeling restriction (FIG. 3). Tables $3 \& 4$ briefly summarize the current therapeutic strategies against CAFs investigated in clinical and preclinical studies. In addition to immune checkpoint molecule-targeted inhibitors such as ipilimumab, pembrolizumab and nivolumab [294], CAFspecific therapies have been an essential complement to immunotherapies and have provided considerable clinical benefits for patients with tumors. However, due to the lack of specific markers for CAFs, as mentioned earlier [120], current CAF-targeting therapies have to address the intractable problem of how to improve the antitumor effect and decrease systematic side effects at the same time, and this issue might explain why only a few CAFtargeted therapies have been translated into the clinic. To discover more specific and efficient molecular targets for CAFs, further in-depth investigations on these cells are still required in the future.

(See figure on next page.)

Fig. 3 Major CAF-targeted immunotherapeutic strategies. There are three main approaches against cancer-associated fibroblasts (CAFs) and their associated molecules for immunotherapy: A Through the immunotherapies or transgenic technologies that targeting CAF markers such as fibroblast activation protein (FAP), alpha-smooth muscle actin (a-SMA) and platelet-derived growth factor receptors (PDGFR), CAFs can be directly depleted and consequently enhance immune response in the tumor microenvironment (TME); B CAF activation and function can be suppressed by inhibiting their crucial effector molecule or signaling pathways such as vitamin A, transforming growth factor-beta (TGF- $\beta$ ), interleukin-6 (IL-6) together with Janus kinase-signal transducer and activator of transcription 3 (JAK-STAT3) signaling pathway, C-C chemokine ligand 2 (CCL2)-C-C chemokine receptor (CCR2) signaling axis and C-X-C chemokine ligand 12 (CXCL12), thereby restricting the immune suppression induced by CAFs in the TME; C CAF-derived matrix proteins such as tenascin-C (TNC), hyaluronan (HA) and matrix metalloproteinases (MMPs) as well as related fibrosis-activated signaling pathways, like focal adhesion kinase (FAK) signaling pathways, are the ideal targets to effectively restrict extracellular matrix (ECM) remodeling 


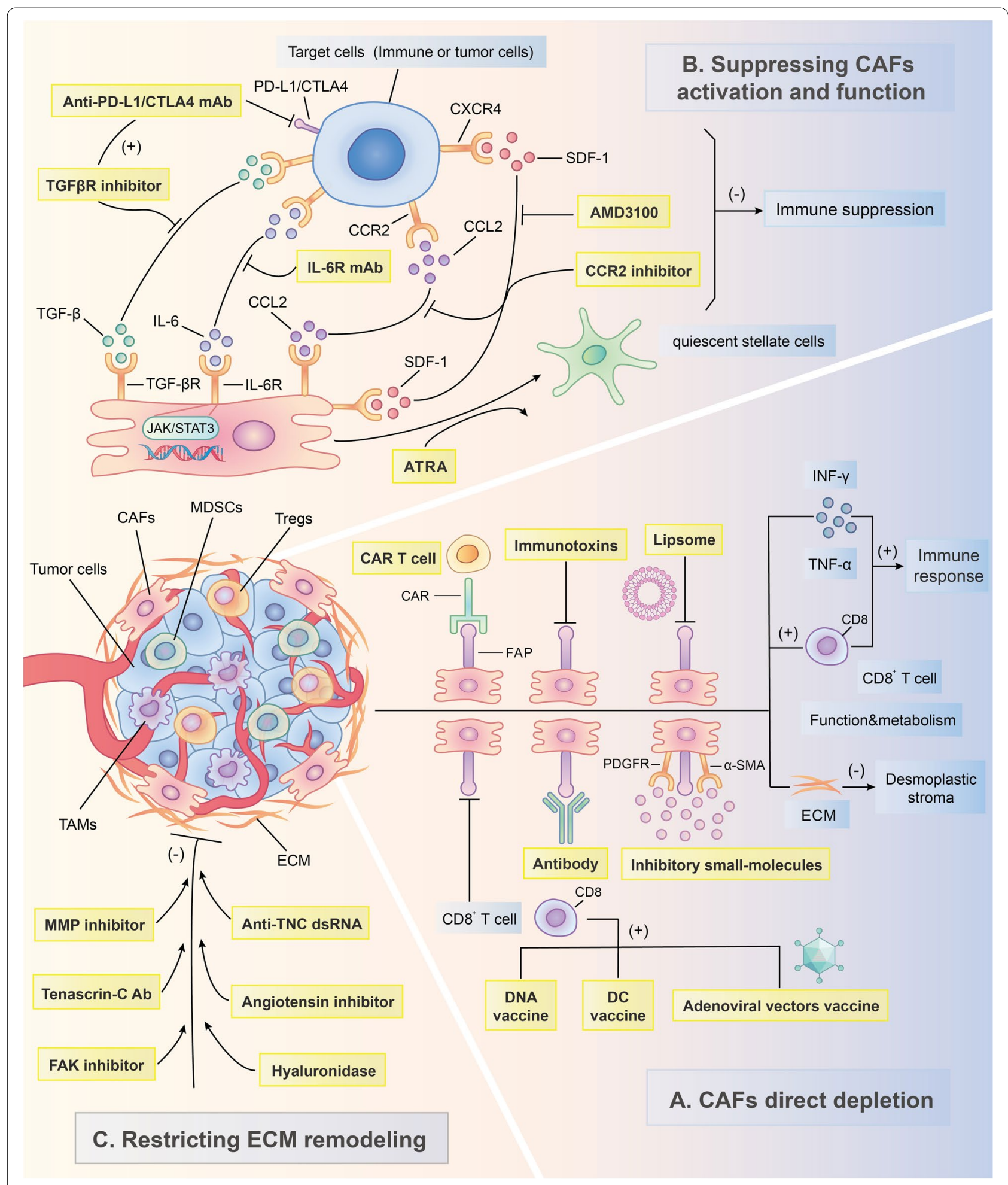

Fig. 3 (See legend on previous page.)

\section{Depleting CAFs directly by targeting surface markers}

Direct CAF-depleting therapeutic strategies mainly depend on surface markers of CAFs, such as FAP, $\alpha$-SMA and PDGFR. Therefore, CAF marker-based inhibitors are currently the major type of CAF-depleting therapies (Table 3). As one of the most viable CAF markers 
Table 3 Multiple preclinical or clinical studies and related drugs for CAF-targeted depletion immunotherapy

\begin{tabular}{|c|c|c|c|c|c|c|}
\hline Drugs & Classification & $\begin{array}{l}\text { Combination } \\
\text { therapy }\end{array}$ & therapeutic effects & Cancer models & Preclinical/Clinical & Refs \\
\hline SynCon DNA vaccine & $\begin{array}{l}\text { FAP-targeted DNA } \\
\text { vaccine }\end{array}$ & $\begin{array}{l}\text { Tumor antigen- } \\
\text { specific DNA vaccine, } \\
\text { Cyclo-phosphamide }\end{array}$ & $\begin{array}{l}\text { Breaks immune toler- } \\
\text { ance and promotes } \\
\text { antitumor immunity }\end{array}$ & $\begin{array}{l}\text { Lung and breast } \\
\text { cancer }\end{array}$ & Preclinical & [295-297] \\
\hline DC-shA20-FAP-TRP2 & $\begin{array}{l}\text { FAP-targeted DC vac- } \\
\text { cine compound }\end{array}$ & Anti-CAFs therapies & $\begin{array}{l}\text { Elicits broad-based } \\
\text { T-cell responses and } \\
\text { anticancer activities }\end{array}$ & Melanoma & Preclinical & {$[298,299]$} \\
\hline AdC68-mFAP vaccine & $\begin{array}{l}\text { FAP-targeted adeno- } \\
\text { viral vectors vaccines }\end{array}$ & None & $\begin{array}{l}\text { Enhances T-cell } \\
\text { responses and inhib- } \\
\text { its tumor proliferation }\end{array}$ & Melanoma & Preclinical & {$[300]$} \\
\hline $\begin{array}{l}\text { FAP-specific CART } \\
\text { cells }\end{array}$ & $\begin{array}{l}\text { FAP-targeted adop- } \\
\text { tive T cell therapy }\end{array}$ & Gemcitabine & $\begin{array}{l}\text { Enhances antitumor } \\
\text { immune responses } \\
\text { and restricts tumor } \\
\text { proliferation, } \\
\text { angio-genesis, ECM } \\
\text { remodeling and } \\
\text { chemoresistance }\end{array}$ & $\begin{array}{l}\text { Pancreatic and lung } \\
\text { cancer }\end{array}$ & Preclinical & [301] \\
\hline $\begin{array}{l}\text { ASGPAGPA-A12ADT/ } \\
\text { DSGETGP-A12ADT }\end{array}$ & $\begin{array}{l}\text { FAP-activated prod- } \\
\text { rugs }\end{array}$ & Thapsigargin & $\begin{array}{l}\text { Enhances the specific } \\
\text { antitumor effect } \\
\text { of drugs with less } \\
\text { systemic toxicity }\end{array}$ & $\begin{array}{l}\text { Breast and prostate } \\
\text { cancer }\end{array}$ & Preclinical & {$[302]$} \\
\hline $\begin{array}{l}\text { Val-boroPro (tala- } \\
\text { bostat) }\end{array}$ & $\begin{array}{l}\text { FAP-targeted inhibi- } \\
\text { tory small-molecules }\end{array}$ & Cisplatin & $\begin{array}{l}\text { Suppresses tumor } \\
\text { growth and inva- } \\
\text { sion and prolongs } \\
\text { patients'survival }\end{array}$ & $\begin{array}{l}\text { Colorectal cancer, } \\
\text { Melanoma }\end{array}$ & Phase II & {$[303,304]$} \\
\hline $\begin{array}{l}\text { RG7386 (FAP-DR5 } \\
\text { Antibody) }\end{array}$ & $\begin{array}{l}\text { FAP-targeted inhibi- } \\
\text { tory antibody }\end{array}$ & $\begin{array}{l}\text { Irinotecan/ Doxoru- } \\
\text { bicin }\end{array}$ & $\begin{array}{l}\text { Induces cell-apop- } \\
\text { tosis and enhances } \\
\text { antitumor immune } \\
\text { responses }\end{array}$ & $\begin{array}{l}\text { Lung, renal, colorec- } \\
\text { tal, and breast cancer }\end{array}$ & Preclinical & {$[305]$} \\
\hline aFAP-PE38 & $\begin{array}{l}\text { FAP-targeted inhibi- } \\
\text { tory immunotoxins }\end{array}$ & $\begin{array}{l}\text { Paclitaxel, Anti-CAF } \\
\text { vaccine }\end{array}$ & $\begin{array}{l}\text { Inhibits tumor angio- } \\
\text { genesis and increases } \\
\text { antitumor activities }\end{array}$ & Breast Cancer & Preclinical & {$[306,307]$} \\
\hline $\begin{array}{l}\text { Bispecific scFv'FAP/ } \\
\text { CD105-IL liposomes }\end{array}$ & $\begin{array}{l}\text { FAP-targeted com- } \\
\text { pound liposomes }\end{array}$ & $\begin{array}{l}\text { Doxorubicin/Trastu- } \\
\text { zumab }\end{array}$ & $\begin{array}{l}\text { Enhances the } \\
\text { cytotoxicity of } \\
\text { Doxorubicin and cell } \\
\text { interaction }\end{array}$ & Fibrosarcoma & Preclinical & [308] \\
\hline $\begin{array}{l}\text { Cellax (Docetaxel- } \\
\text { conjugate nanopar- } \\
\text { ticles) }\end{array}$ & $\begin{array}{l}\text { a-SMA-targeted } \\
\text { nanoparticles }\end{array}$ & None & $\begin{array}{l}\text { Enhances anti-stro- } \\
\text { mal effect and inhib- } \\
\text { its tumor metastasis } \\
\text { and angiogenesis }\end{array}$ & $\begin{array}{l}\text { PDAC and breast } \\
\text { cancer }\end{array}$ & Preclinical & {$[309,310]$} \\
\hline Crenolanib & $\begin{array}{l}\text { PDGFR-targeted } \\
\text { inhibitor }\end{array}$ & None & Not available & $\begin{array}{l}\text { Gastro-intestinal } \\
\text { stromal tumor }\end{array}$ & Phase III & [311] \\
\hline
\end{tabular}

Diverse CAF-targeted depleting immunotherapeutic strategies in preclinical/clinical studies

FAP fibroblast activation protein, DC dendritic cell, shA20 A20-specific shRNA, TRP2 tyrosine-related protein 2, AdC68 adenoviral vector of chimpanzee serotype 68 , CAR $T$ cells chimeric antigen receptor T cells, CAFs cancer-associated fibroblasts, $E C M$ extracellular matrix, $D R 5$ death receptor $5, P E 38$ a genetically engineered form of the Pseudomonas exotoxin, sCFV single chain antibody fragment, CD105 cluster of differentiation $105, I L$ interleukin, $a$-SMA alpha smooth muscle actin, PDAC pancreatic ductal adenocarcinoma, PDGFR platelet-derived growth factor receptor

for potential clinical application, FAP has been prominent in recent studies of CAF-targeted therapy [324, 325]. The elimination of CAFs by FAP-targeting therapy can enhance the anticancer immune response mediated by high levels of certain inflammatory mediators, such as IFN- $\gamma$ and TNF- $\alpha$, and facilitate the toxic effects and metabolism of CD8 $+\mathrm{T}$ cells with a decreased desmoplastic stroma in the TME [224, 300, 301, 326]. Current FAP-targeting therapies mainly include diverse types of tumor vaccines and other immunotherapeutic approaches, such as adoptive T cell therapy, all of which can eliminate FAP + cells [327, 328].

FAP-based DNA vaccines are one of the principal types of cancer vaccines $[315,329]$. The first DNA vaccine against the cancer stromal antigen FAP was developed by Loeffler et al. [330] in multidrug-resistant colon and breast carcinoma murine models. This vaccine is capable of eliminating CAFs by stimulating a CD8 $+\mathrm{T}$ 
Table 4 Diverse designed drugs that potentially target CAF-associated effector molecules, signaling pathways and matrix proteins

\begin{tabular}{|c|c|c|c|c|c|c|}
\hline Drugs & Mechanisms & Combination therapy & therapeutic effects & Cancer models & Status & Refs \\
\hline $\begin{array}{l}\text { All-trans retinoic acid } \\
\text { (ATRA) }\end{array}$ & $\begin{array}{l}\text { Retinol levels restora- } \\
\text { tion, PSC de-activation }\end{array}$ & None & $\begin{array}{l}\text { Increases T-cell infiltra- } \\
\text { tion and Inhibits tumor } \\
\text { growth and invasion }\end{array}$ & PDAC & Preclinical & {$[312]$} \\
\hline Galunisertib & TGF- $\beta R 1$ inhibition & Gemcitabine & $\begin{array}{l}\text { Prolongs patients' } \\
\text { survival with minimal } \\
\text { added toxicity }\end{array}$ & $\begin{array}{l}\text { Pancreatic and hepato- } \\
\text { cellular cancer }\end{array}$ & Phase II & {$[313,314]$} \\
\hline $\begin{array}{l}\text { Anti-CTLA4-TGF- } \beta R 2 / \\
\text { Anti-PD-L1-TGF- } \beta \text { R2 }\end{array}$ & $\begin{array}{l}\text { TGF- } \beta R 2 \text { and immune } \\
\text { checkpoints inhibition }\end{array}$ & None & $\begin{array}{l}\text { Decreases tumor- } \\
\text { infiltrating Tregs and } \\
\text { suppresses tumor } \\
\text { progression }\end{array}$ & Breast cancer & Preclinical & {$[315]$} \\
\hline $\begin{array}{l}\text { Tocilizumab (monoclo- } \\
\text { nal antibody) }\end{array}$ & $\begin{array}{l}\text { IL-6-JAK/STAT3 signaling } \\
\text { pathway inhibition }\end{array}$ & $\begin{array}{l}\text { Carboplatin/Doxoru- } \\
\text { bicin }\end{array}$ & $\begin{array}{l}\text { Enhances antitumor } \\
\text { immunity and provides } \\
\text { survival benefits }\end{array}$ & $\begin{array}{l}\text { Recurrent epithelial } \\
\text { ovarian cancer }\end{array}$ & Phase I & {$[316]$} \\
\hline CCX872 & $\begin{array}{l}\text { CCL2-CCR2 signaling } \\
\text { axis inhibition }\end{array}$ & $\begin{array}{l}\text { FOLFIRINOX (fluoroura- } \\
\text { cil, leucovorin, irinote- } \\
\text { can and oxaliplatin) }\end{array}$ & $\begin{array}{l}\text { Restricts immune sup- } \\
\text { pression and improves } \\
\text { clinical prognosis }\end{array}$ & Pancreatic cancer & Phase I & {$[317,318]$} \\
\hline AMD3100 & CCR4 inhibition & Anti-PD-L1 therapy & $\begin{array}{l}\text { Promotes T-cell } \\
\text { accumulation and } \\
\text { eliminates cancer cells }\end{array}$ & Pancreatic cancer & Preclinical & [114] \\
\hline F16-IL-2 & $\begin{array}{l}\text { Tenascin- } \mathrm{C} \text { depletion } \\
\text { and IL-2 delivery }\end{array}$ & Paclitaxel/ Doxorubicin & $\begin{array}{l}\text { Enhances antitumor } \\
\text { immunity and inhibits } \\
\text { tumor growth }\end{array}$ & Breast cancer & Preclinical & [319] \\
\hline VS-4718 (FAK inhibitor) & FAK-targeted inhibition & Anti-PD-1 therapy & $\begin{array}{l}\text { Inhibits the infiltration } \\
\text { of immuno-suppressive } \\
\text { cells and improves } \\
\text { survival }\end{array}$ & $\begin{array}{l}\text { Non-small-cell lung } \\
\text { cancer, mesothelioma } \\
\text { and pancreatic neo- } \\
\text { plasms }\end{array}$ & Phase I & [320] \\
\hline $\begin{array}{l}\text { anti-TNC dsRNA (ATN- } \\
\text { RNA) }\end{array}$ & $\begin{array}{l}\text { Tenascin-C mRNA- } \\
\text { targeted interference }\end{array}$ & Surgery & $\begin{array}{l}\text { Prolongs patients'sur- } \\
\text { vival and restricts tumor } \\
\text { recurrence }\end{array}$ & $\begin{array}{l}\text { Brain glioblastoma } \\
\text { multiforme }\end{array}$ & Phase I & [321] \\
\hline PEGPH2O & $\begin{array}{l}\text { Tumor stromal } \\
\text { hyaluronan-targeted } \\
\text { depletion }\end{array}$ & $\begin{array}{l}\text { Gemcitabine and nab- } \\
\text { paclitaxel }\end{array}$ & $\begin{array}{l}\text { Prolongs patients' } \\
\text { survival with less sys- } \\
\text { tematic side effect }\end{array}$ & PDAC & Phase III & [322] \\
\hline $\begin{array}{l}\text { Losartan (angiotensin } \\
\text { inhibitor) }\end{array}$ & $\begin{array}{l}\text { Profibrotic signals } \\
\text { inhibition }\end{array}$ & None & $\begin{array}{l}\text { Facilitates drugs deliv- } \\
\text { ery and restricts ECM } \\
\text { remodeling }\end{array}$ & $\begin{array}{l}\text { Pancreatic and breast } \\
\text { cancer }\end{array}$ & Preclinical & [323] \\
\hline
\end{tabular}

Diverse strategies in CAF-targeted immunotherapies that suppress CAF activation and function and restrict ECM remodeling

CAF cancer-associated fibroblast, PSC pancreatic stellate cell, PDAC pancreatic ductal adenocarcinoma, TGF- $\beta R 1$ transforming growth factor beta receptor $1, T G F-\beta R 2$ transforming growth factor beta receptor 2, CTLA-4 cytotoxic lymphocyte-associated antigen-4, PD-L1 programmed death ligand 1, IL-6 interleukin-6, JAK Janus kinase, STAT3 signal transducer and activator of transcription 3, CCX872 one of CCR2 antagonists, CCL2 C-C chemokine ligand 2, CCR2 C-C chemokine receptor 2, AMD3100 one of CXCR4 antagonists, CCR4 C-C chemokine receptor 4, IL-2 interleukin-2, FAK focal adhesion kinase, PD-1 programmed cell death protein 1, TNC tenascin-C, PEGPH20 a PEGylated human recombinant $\mathrm{PH} 20$ hyaluronidase, ECM extracellular matrix

cell-mediated immune response and further inhibit tumor growth and metastasis [330]. Recently, with advances in DNA vaccine studies, a novel type of vaccine termed the SynCon FAP DNA vaccine has been shown to not only disrupt immune tolerance and promote the antitumor immunity of both $\mathrm{CD} 8+$ and $\mathrm{CD} 4+\mathrm{T}$ cells but also enhance the effects of other relevant tumor antigen-specific DNA vaccines [295]. Since the anticancer therapeutic effect of a single DNA vaccine targeting FAP is extremely limited [331], subsequent studies identified a novel therapeutic strategy that combines cyclophosphamide (CY) with a DNA vaccine that significantly increases the tumor inhibition rate by overcoming the tumor-stromal blockade and enhancing the nonspecific toxic effects of CY on tumor cells [296, 297].
In addition, DC vaccines are regarded as an effective strategy that induces a strong tumor immune response by replacing the role of impaired DCs in the TME [332]. Specifically, DC vaccines can enhance tumor antigen presentation by increasing costimulatory molecule and proinflammatory cytokine expression, thereby heightening cancer-specific $\mathrm{T}$ cell responses [333]. To improve the finite therapeutic effect of the previously established A20-silenced DC vaccine, Gottschalk et al. [298] developed a compound DC vaccine (DC-shA20-FAP-TRP2) that cotargets both tumor cells and FAP-positive CAFs. This vaccine was reported to elicit broad $\mathrm{T}$ cell responses and potent antitumor activity [298]. Moreover, when cooperating with other anti-CAF therapies, DC-based vaccines have been shown to reduce the level of TGF- $\beta$ 
and consequently inhibit the migration of Treg cells into tumors [299]. Recently, studies have demonstrated that the fusion of DCs and CAFs contributes to a strong CTL response against CAFs, suggesting that it might be a potential method for improving the anticancer effect of current DC vaccine strategies [334]. Adenoviral vector vaccines are another type of FAP-targeting vaccine [335]. Similar to DNA vaccines, adenoviral vector vaccines such as the adenoviral vector of chimpanzee serotype 68 (AdC68)-mFAP vaccine can also induce $\mathrm{T}$ cell recruitment and enhance the function of melanoma-specific effector CD8 $+\mathrm{T}$ cells, thereby destroying FAP + stromal cells within the TME [300]. Additionally, other tumor vaccines contain whole-cell vaccines $[312,336]$ and peptide immunization vaccines [337].

FAP is also an important target for adoptive $\mathrm{T}$ cell therapy, especially for chimeric antigen receptor (CAR) therapy [338]. FAP-specific CAR T cells function to deplete most FAP + cells and restrict tumor stroma generation, along with promoting the uptake and antitumor effects of chemotherapeutic drugs, such as gemcitabine [301]. Notably, several studies have observed that the elimination of FAP + cells by CAR T cells causes severe side effects, such as significant bone marrow toxicity and cachexia [339, 340]. Considering that CAR T cells usually deplete FAP-overexpressing cells (e.g., CAFs) rather than normal cells with basal FAP levels, there might exist a different window of therapeutic opportunity for differential single-chain variable fragments (scFv) of CAR constructs [326]. In view of the possible toxicity of FAP-targeted adoptive $\mathrm{T}$ cell therapy, scientists try to develop prodrugs that are activated only by FAP through unique postprolyl endopeptidase activity, and these prodrugs have been proven to induce less systemic toxicity and have greater therapeutic potential [341, 342]. For instance, an in vivo and in vitro study in breast and prostate cancer illustrated that a FAP-activated prodrug contributed to the selective death of stromal cells and exerted a significant anticancer effect [302]. Finally, other FAP-targeting treatments, including FAP-inhibiting small molecules (talabostat and cisplatin) [303, 304], antibodies [305], immunotoxins [306, 307] and FAP-targeted liposomes [308, 343], also provide therapeutic benefits.

$\alpha$-SMA has been identified as another surface marker of CAFs [324]. Current studies of therapies targeting $\alpha$-SMA remain stagnant because of their dual effects on tumor progression. First, the depletion of $\alpha$-SMA + CAFs was proven to suppress the metastasis of cancer cells as well as tumor angiogenesis in breast cancer and PDAC models [309, 310]. However, more importantly, targeting $\alpha$-SMA was also reported to induce disease aggression and progression by enhancing the infiltration of CD3 + Foxp3 + Treg cells in the TME [310]. For other CAF markers, such as PDGFR, the associated clinical trials are still ongoing [311, 344].

As discussed before, neither FAP nor $\alpha$-SMA is exclusively expressed on CAFs, suggesting that more highly selective markers are required to improve the precision of CAF-based therapies. Recently, Su et al. [104] identified two novel specific surface proteins for a CAF subpopulation (CD10 and GPR77), which might be promising targets for inhibiting tumorigenesis and tumor chemoresistance.

\section{Suppressing CAF activation and function by targeting associated effector molecules}

Considering the crucial role of interactions between CAFs and other cells, particularly the crosstalk between CAFs and the TIME, in the immune suppression induction of the TME, it seems more feasible to restrict CAF activation and their interacting progress by targeting CAF-associated crucial effector molecules such as growth factors, cytokines and chemokines as well as signaling pathways (Table 4). For example, vitamin A deficiency is a main contributor to the activation of PSCs [43]. Therefore, by restoring retinol levels in PSCs, alltrans retinoic acid (ATRA) can reset them to the inactive state [43]. In a parallel study, ATRA treatment of a PDAC model exerted substantial antitumor effects, including remarkably increasing the numbers of CD8 $+\mathrm{T}$ cells in juxta-tumoral compartments and limiting tumor cell invasion [312]. TGF- $\beta$ plays an important role in the activation of CAFs and the interaction between CAFs and immune cells, as previously described, indicating that TGF- $\beta$ inhibition therapy might be capable of restoring impaired immune responses in the TME [30, 191, 213]. Currently, multiple preclinical and clinical studies of TGF- $\beta$-based immunotherapies are ongoing [192]. Galunisertib (LY21577299), for example, is a small-molecule inhibitor of transforming growth factor beta receptor 1 (TGF- $\beta$ R1) with discernable cardiac toxicities rarely reported during treatment [345]. Phase II clinical trials for pancreatic cancer and hepatocellular carcinoma have exhibited the significant therapeutic activity of galunisertib against tumors, whether administered in combination with gemcitabine or as monotherapy [313, 314]. Additional reports have documented that the combination of a treatment targeting CAF-derived TGF- $\beta$ with checkpoint inhibitors such as anti-PD-L1 antibodies exerts greater immunological effects on tumors than the respective monotherapies [346-348]. Therefore, Ravi et al. [349] attempted to engineer anti-CTLA4 or anti-PD-L1 antibodies fused with the TGF- $\beta$ R2 extracellular domain, resulting in anti-CTLA4-TGF- $\beta$ R2 and 
anti-PDL1-TGF- $\beta$ R2 chimeras. Compared with ipilimumab (a type of anti-CTLA-4 antibody) monotherapy, the anti-CTLA4-TGF- $\beta$ R2 molecule presents more effective at decreasing tumor-infiltrating Treg cells and suppressing tumor progression [315]. In addition, previous studies have demonstrated that IL-6 together with the JAK/STAT3 signaling pathway in the TME participate in processes that strongly suppress immune effector cell function and facilitate tumor progression induced by CAFs [122, 243, 350-352]. Tocilizumab, a humanized anti-IL-6R monoclonal antibody, has exhibited extensive antitumor and anti-chemoresistance effects on multiple cancer types in preclinical studies [353-355]. In a phase I clinical trial, high-dose tocilizumab was observed to stimulate $\mathrm{CD} 8+\mathrm{T}$ cell activation and increase the levels of antitumor-associated effectors such as IFN- $\gamma$ and TNF- $\alpha$, thereby enhancing anticancer immunity [316]. Moreover, preclinical evidence indicates that therapy targeting IL-6/JAK/STAT3 signaling might also augment the antitumor efficacy of immune checkpoint-inhibiting monoclonal antibodies [356, 357]. Since the CCL2-CCR2 signaling axis plays an essential role in MDSC-induced immune suppression, therapies suppressing the CCL2CCR2 signaling pathway might be effective in blunting MDSC immunoinhibitory effects [242]. Clinical trials have previously reported that CCR2 inhibition in combination with FOLFIRINOX (fluorouracil[5-FU], leucovorin, irinotecan and oxaliplatin) can significantly reduce the numbers of tumor-infiltrating macrophages and Treg cells while increasing the number of effector $\mathrm{T}$ lymphocytes in the TME, consequently enhancing antitumor immunity in pancreatic cancer [358]. Recently, a treatment combining CCX872 (a CCR2-specific antagonist) and FOLFIRINOX was reported to achieve a better therapeutic effect and clinical prognosis with less M-MDSC infiltration [317, 318]. Another essential chemokine, SDF-1 (also termed CXCL12), is also involved in the activation and immune suppression of CAFs. Via blocking the combination of SDF-1 and its receptor CXCR4, AMD3100 (a CXCR4 inhibitor) is able to rapidly promote the accumulation of $\mathrm{T}$ cells and effectively eliminate cancer cells by synergizing with an anti-PD-L1 antibody [114].

\section{Restricting CAF-induced ECM remodeling in the TME}

CAF-targeted treatments are also being designed to block fibrosis progression, including therapies targeting fibrosis-activated signaling pathways and their fibrosis products (Table 4), which ultimately restrict CAF-induced ECM remodeling. The altered ECM after treatment partly alleviates the suppression of immune effector cell recruitment into tumors in the TME, thus enhancing anticancer immunity $[281,282,359]$.
As mentioned before, the FAK signaling pathway is an important fibrosis-activated signaling pathway of CAFs involved in matrix stiffness and immune suppression [284]. A specific FAK inhibitor (VS-4718) was reported to inhibit immunosuppressive cell infiltration, such as TAMs, MDSCs and Treg cells, in the TME, and significantly improved overall survival (OS) in a PDAC model [284]. Moreover, as FAK inhibitors might heighten the antitumor effect of immune checkpoint inhibitors, associated phase I clinical trials have been set up to assess their therapy responses [320].

Therapies against CAF-derived ECM proteins, such as tenascin C (TNC), HA and MMPs, might also be capable of inhibiting desmoplastic reactions and consequently reducing the immunosuppressive effect of ECM on immune cells. The ECM protein TNC appears to be an appealing target for antitumor treatment due to its high expression in cancer tissues and functional association with tumor cell adhesion, migration, invasion and proliferation along with immune evasion [323]. Several antibodies have been established to specifically target TNC in order to improve the delivery of effector molecules into TNC-rich tumor tissue [360]. For example, the antibody F16 shows good specificity for TNC and is designed in complex with IL-2 to promote the recruitment of immune cells in the TME [361]. In a breast cancer model, when in combination with F16-IL-2, cytotoxic drugs such as paclitaxel or doxorubicin induce a more obvious restriction of tumor growth than chemotherapeutic agents alone [319]. Furthermore, anti-TNC dsRNA (ATN-RNA), which has sequence homology to TNC mRNA and is developed using RNA-based technologies, has produced substantial improvements in the clinical prognosis of patients with glioblastoma multiforme (GBM) [321]. Recent research in autophagy-deficient TN breast cancer revealed that TNC suppression sensitizes $\mathrm{T}$ cell-mediated cell killing and enhances the anticancer effects of single anti-PD1/PD-L1 therapy, indicating a potential therapeutic strategy that links TNC inhibitors and immune checkpoint blockade (ICB) for TN breast cancer [362]. Excessive tumor-stromal HA together with collagen usually results in substantial vessel compression, which blocks the delivery of peripheral immune cells and drugs into tumor vessels [363]. PEGPH20, a PEGylated human recombinant $\mathrm{PH} 20$ hyaluronidase, functions to deplete HA and then potentiate chemotherapeutic efficiency by improving vascular patency. Further clinical trials confirmed that PEGPH20 along with gemcitabine and nab-paclitaxel combination therapy could suppress tumor growth and significantly increase patient survival [322]. Moreover, losartan (an angiotensin inhibitor) also exhibits the ability to reduce the production of stromal collagen and HA by inhibiting TGF- $\beta 1$, connective tissue 
growth factor (CTGF) and endothelin-1 (ET-1) profibrotic signals [323]. Finally, regarding MMP therapies, the disappointing antitumor effects of current MMP inhibitors have been gradually reported, but several novel types are being translated into early clinical trials [364].

\section{Challenges and directions}

Considering a large number of CAF characteristics might change with the culture environment alteration (in vivo to in vitro), some questions have continuously arisen and need to be solved. First, to retain the CAF phenotype in in vitro culture as much as possible, researchers have tried various culture conditions and found that lower serum concentrations and matrices with more physiological mechanical properties might be preferable to keep the CAF original phenotype [87]. Recent studies have identified that several inhibitors of CAF activating molecules, such as TGF- $\beta$ inhibitors, can effectively restrict the transformation of the CAF phenotype in vitro culture [365], which indicates that adding some CAF activator suppressors into in vivo culture medium might be a novel strategy to accurately preserve the in vivo phenotype of CAFs. Certainly, more in-depth studies are required to investigate more suitable in vitro culture conditions for CAFs.

Second, currently, single-cell transcriptome analyses have been a useful method to understand the characteristics and heterogeneity of CAFs. Aside from singlecell transcriptome analyses, researchers usually utilize immunoassay technology, such as high-quality antibodies against CAF marker proteins, to detect CAFs in tissue. However, due to the heterogeneity of CAFs, antibodies against certain CAF subpopulation markers require complex optimization, which hampers their adoption in laboratories. Recently, the technology of multiplexed mRNA probes has been rapidly developed, and thus accurate quantitative methods for the detection of CAFs, in the long term, are promising [87]. Further investigations should be performed to explore and develop more universal, stable, standardized and accurate quantitative methods for CAF detection in the future.

In addition, while multiple methods have been recently developed for the detection of CAF phenotype expression, as introduced above, such as specific antibodies, mRNA probes and transcriptome analyses, there is still a lack of a method to identify CAF phenotype changes in a timely and precise manner during the cultivation process.

Finally, it is necessary to deepen the understanding regarding the origins and subpopulations of CAFs, especially the time and stage heterogeneity of CAFs [366], by investigating CAFs in different experimental stages and different clinical stages. According to a previous study [367], for example, researchers can perform a longitudinal study of whole CAF populations in certain cancer animal models by utilizing whole transcriptome analysis in FACS-sorted fibroblasts from early to late stages or distinct pathological grades, and ultimately observe alterations in the CAF transcriptome and phenotype. Furthermore, a longitudinal study of certain fibroblast cell lines in different culture stages can also be conducted, including primary, early isolation, long-term passage and immortalization cells, through techniques such as singlecell transcriptome analyses.

Additionally, to date, the origins and subtypes of some CAFs, especially the anticancer subpopulation (rCAFs), are still unknown, and a deep understanding of rCAFs may become a future research direction. Moreover, the number of studies on CAF-immune cell interactions is far from sufficient, and most of the studies mentioned in our review have not completely illustrated the detailed cell-internal mechanisms by which CAFs affect immune cells. These factors should be considered in further subsequent investigations. Therefore, a thorough exploration of the crosstalk between CAFs and the TIME is required in the future to enable us to identify the basis of the impaired immune response induced by CAFs and might identify an essential method to restimulate the antitumor response which is distinct from strategies that directly restrict and eliminate cancer cells.

Furthermore, although an increasing number of CAFtargeting therapeutic strategies are being developed, the lack of more specific markers and the low number of large-scale randomized clinical trials are still two huge challenges facing CAF-targeting treatment.

\section{Conclusions}

Since it acts as a crucial role in tumor initiation and progression in the TME, CAFs have received increasing attention in the past decade. CAF populations exhibit extensive heterogeneity in terms of cell origin and phenotype, which leads to their distinct behaviors during cancer development: most CAF subtypes (pCAFs) function as tumor facilitators; however, some other subtypes (rCAFs) exert tumor-inhibiting effects. Additionally, the intertransformation of several subpopulations partly indicates the plasticity of CAFs, while more investigations are needed to confirm this. Recently, studies have confirmed the importance of the interaction between CAFs and the immune microenvironment in the TME during tumor progression. Meanwhile, an increasing number of research regarding the effect of CAFs on the immune components of the TIME have gradually clarified the mechanisms by which CAFs orchestrate an immunosuppressive TME, and the results facilitate the translation of related CAF-based therapeutic targets into clinical trials.

In this review, we describe the interaction between CAFs and immune cells infiltrating the TME in detail 
and propose a possible immune inhibitory mechanism by which CAFs not only directly influence the activities of immune cells but also indirectly result in immune effector cell dysfunctions by upregulating immune checkpoint molecule expression on the cell surface and remodeling the ECM within the TME. By secreting various chemokines, cytokines and other effector molecules, CAFs directly inhibit immune cell-mediated antitumor immunity mainly through three main mechanisms, as listed below: (i) to drive the abnormal polarization or trans-differentiation of immune cells such as TAMs, TANs, MCs, DCs and T lymphocytes into certain procancerogenic cell subsets; (ii) to promote the activities of immune inhibitory cells, including M2-type TAMs, N2-type TANs, rDCs, Treg cells and MDSCs, in terms of their recruitment, infiltration, activation and immunosuppressive behaviors; and (iii) to reduce the cytotoxic activities and cytokine secretion of immune effector cells like NK cells and CTLs. Notably, some infiltrating immune cells, such as TAMs, TANs, MCs and DCs, are capable of enhancing the activation and function of CAFs. These interactions constitute immunoinhibitory loops that further heighten immune suppression in the TME. Moreover, CAFs have also been reported to modulate anticancer immunity through indirect means: (i) to upregulate the expression of immune checkpoint molecules such as PD-1/PD-L1 in both themselves and other cells in the TME to induce $\mathrm{T}$ cell dysfunction and immunologic tolerance; (ii) to degrade and remodel the ECM through the production of fibronectin, collagen and MMPs and the activation of FAK to restrict effector immune cell infiltration while increasing the recruitment of inhibitory immune cells, such as Tregs, MDSCs and TAMs, and consequently block the initiation of the immune response. Certainly, excess expression of immune checkpoints in CAFs and surrounding matrix deposition would in turn prolong CAF survival, stimulating their activation and maintaining their protumor properties. In view of the diverse immune suppressive effects of CAFs, current CAF-targeted therapeutic strategies that target CAF surface markers, associated effector molecules and their relevant signaling pathways along with restricted ECM remodeling have been developed to enhance antitumor immunity, which has produced considerable clinical benefits. More importantly, in combination with checkpoint blockade immunotherapies or chemotherapies, CAF-targeted treatment might hold promise for the treatment of tumors with a fibroblast-rich TME.

\section{Abbreviations}

CAFs: Cancer-associated fibroblasts; TME: Tumor microenvironment; ECM: Extracellular matrix; TIME: Tumor immune microenvironment; $a-S M A$ : Alpha smooth muscle actin; FAP: Fibroblast activation protein; FSP1:
Fibroblast-specific protein 1; PDGFR: Platelet-derived growth factor receptor; CD: Cluster of differentiation; MMP: Matrix metalloproteinase; Treg: T regulatory; MDSCs: Myeloid-derived suppressor cells; IL: Interleukin; TGF: Transforming growth factor; HGF: Hepatocyte growth factor; PDGF: Platelet-derived growth factor; FGF-2: Fibroblast growth factor 2; SDF-1: Stromal-derived factor-1; ROS: Reactive oxygen species; PSCs: Pancreatic stellate cells; HSCs: Hepatic stellate cells; ISCs: Islet stellate cells; IGF-1: Insulin-like growth factor-1; MSCs: Mesenchymal stem cells; BMSCs: Bone marrow mesenchymal stem cells; EMT: Epithelial-mesenchymal transition; CXCL: C-X-C chemokine ligand; MZF1: Myeloid zinc finger 1; CCL: C-C chemokine ligand; ER: Endoplasmic reticulum; HASCs: Human adipose tissue-derived stem cells; ADFs: Adipocytederived fibroblasts; EndMT: Endothelial-to-mesenchymal transition; MMT: Mesothelial-mesenchymal transition; MMT: Monocyte-to-myofibroblast transdifferentiation; MAPK: P38-mitogen-activated protein kinase; JAK: Janus kinase; STAT3: Signal transducer and activator of transcription 3; DAMPs: Damageassociated molecular patterns; NLRP3: NOD-like receptor protein 3; HSF1: Heat shock factor 1; YAP: Yes-associated protein; TAZ: Tafazzin; DKK3: Dickkopf-3; LIF: Leukemia inhibitory factor; SHP-1: SH2-containing protein tyrosine phosphatase-1; PDAC: Pancreatic ductal adenocarcinoma; myCAFs: Myofibroblastic CAFs; iCAFs: Inflammatory CAFs; apCAFs: Antigen-presenting CAFs; MHC:

Major histocompatibility complex; FB: Fibroblast population; POSTN: Periostin; MYH11: Myosin-11; PDPN: Podoplanin; CAV1: Caveolin 1; OSCC: Oral squamous cell carcinoma; HGSOC: High-grade serous ovarian cancer; pCAFs: Cancerpromoting CAFs; rCAFs: Cancer-restraining CAFs; BMP-4: Bone morphogenetic protein 4; EGFR: Epidermal growth factor receptor; TAMs: Tumor-associated macrophages; LPS: Lipopolysaccharide; TNF: Tumor necrosis factor; S100A4: S100 calcium binding protein A4; MCP-1: Monocyte chemotactic protein-1; Chi3L1: Chitinase 3-like 1; PD-1: Programmed cell death protein 1; M-CSF1: Macrophage colony-stimulating factor 1; ERa: Estrogen receptor alpha; SR-A: Class A scavenger receptors; TANs: Tumor-associated neutrophils; CLCF1: CAFderived cardiotrophin-like cytokine factor 1; PD-L1: Programmed death ligand 1; CXCR: C-X-C chemokine receptor; GC-MSCs: Gastric cancer-mesenchymal stem cells; ERK1/2: Extracellular regulated protein kinases 1/2; MCs: Mast cells; VEGF: Vascular endothelial growth factor; SCF: Stem cell factor; NK: Natural killer; NKG: NK group; DNAM-1: DNAX accessory molecule 1; KIRs: Killer immunoglobulin-like receptors; PGE2: Prostaglandin E2; IDO: Indoleamine 2,3-dioxygenase; DAP12: DNAX-activation protein 12; IFN- $\gamma$ : Interferon- $\gamma$; MICA/B: MHC class I chain-related gene A/B; PVR: Poliovirus receptor; DCs: Dendritic cells; rDCs: Regulatory DCs; TDO2: Tryptophan 2,3-dioxygenase; Th: Helper T; CTLs: Cytotoxic T lymphocytes; DPP4: Dipeptidyl peptidase IV; TSLP: Thymic stromal lymphopoietin; TLR8: Toll-like receptor 8; ICAM: Intercellular cell adhesion molecule; VCAM-1: Vascular cell adhesion molecule-1; HIC-5: Hydrogen peroxide inducible clone-5; TCR: T cell receptor; FAS: Factor associated suicide; FASL: Factor associated suicide ligand; PD-L2: Programmed death ligand 2; PMNMDSCs: Polymorphonuclear MDSCs; M-MDSCs: Monocytic MDSCs; NO: Nitric oxide; LSCC: Lung squamous cell carcinoma; CCR: C-C chemokine receptor; TN: Triple-negative; GACs: Gastric adenocarcinomas; CTLA-4: Cytotoxic lymphocyte-associated antigen-4; TIM-3: Mucin-domain containing-3; LAG-3: Lymphocyte-activation gene-3; PI3K: Phosphatidylinositol 3-kinase; AKT(PKB): Protein kinase B; ERK5: Extracellular signal regulated kinase 5; ANLN: Anillin; DIAPH3: Diaphanous-related formin-3; SFKs: Src family kinases; FAK: Focal adhesion kinase; HA: Hyaluronan; CY: Cyclophosphamide; shA20: A20-specific shRNA; TRP2: Tyrosine-related protein 2; AdC68: Adenoviral vector of chimpanzee serotype 68; CAR: Chimeric antigen receptor; ScFv: Single-chain variable fragments; TGF- $\beta R$ : Transforming growth factor beta receptor; TNC: Tenascin C; ATN-RNA: Anti-tenascin C dsRNA; ICB: Immune checkpoint blockade; CTGF: Connective tissue growth factor; ET-1: Endothelin-1.

\section{Acknowledgements}

Not applicable.

\section{Authors' contributions}

XQM, JX and WW collected the related studies and drafted the manuscript. $\mathrm{CL}, J \mathrm{H}$, JL and BZ participated in the design of the review. SS, XJY and QCM initiated the study and revised the manuscript. All authors read and approved the final manuscript.

\section{Funding}

This study was jointly funded by the National Natural Science Foundation of China (No. 81802352, 81772555 and 81902428), the National Science 
Foundation for Distinguished Young Scholars of China (No. 81625016), the Shanghai Sailing Program (No. 19YF1409400 and 20YF1409000), the Shanghai Rising-Star Program (No. 20QA1402100), the Shanghai Anticancer Association Young Eagle Program (No. SACA-CY19A06), the Clinical and Scientific Innovation Project of Shanghai Hospital Development Center (No. SHDC12018109 and SHDC12019109) and the Scientific Innovation Project of Shanghai Education Committee (No. 2019-01-07-00-07-E00057).

\section{Availability of data and materials}

Not applicable.

\section{Declarations}

Ethics approval and consent to participate

Not applicable.

\section{Consent for publication}

Not applicable.

\section{Competing interests}

The authors declare that they have no competing interests.

\section{Author details}

${ }^{1}$ Department of Pancreatic Surgery, Fudan University Shanghai Cancer Center, No. 270 Dong'An Road, Xuhui District, Shanghai 200032, China. ${ }^{2}$ Department of Oncology, Shanghai Medical College, Fudan University, Shanghai 200032, China. ${ }^{3}$ Shanghai Pancreatic Cancer Institute, Shanghai 200032, China. ${ }^{4}$ Pancreatic Cancer Institute, Fudan University, Shanghai 200032, China.

Received: 1 July 2021 Accepted: 11 September 2021

Published online: 11 October 2021

\section{References}

1. Chen F, et al. New horizons in tumor microenvironment biology: challenges and opportunities. BMC Med. 2015;13:45.

2. Paluskievicz CM, et al. T regulatory cells and priming the suppressive tumor microenvironment. Front Immunol. 2019;10:2453.

3. Quail DF, Joyce JA. Microenvironmental regulation of tumor progression and metastasis. Nat Med. 2013;1911:1423-37.

4. Schulz M, Salamero-Boix A, Niesel K, Alekseeva T, Sevenich L. Microenvironmental regulation of tumor progression and therapeutic response in brain metastasis. Front Immunol. 2019;10:1713.

5. Giraldo NA, et al. The clinical role of the TME in solid cancer. Br J Cancer. 2019;1201:45-53.

6. Li H, Fan X, Houghton J. Tumor microenvironment: the role of the tumor stroma in cancer. J Cell Biochem. 2007;1014:805-15.

7. Liu T, et al. Cancer-associated fibroblasts: an emerging target of anticancer immunotherapy. J Hematol Oncol. 2019;121:86.

8. Bu L, et al. Biological heterogeneity and versatility of cancerassociated fibroblasts in the tumor microenvironment. Oncogene. 2019;3825:4887-901.

9. Chen X, Song E. Turning foes to friends: targeting cancer-associated fibroblasts. Nat Rev Drug Discov. 2019;182:99-115.

10. Mizutani Y, et al. Meflin-positive cancer-associated fibroblasts inhibit pancreatic carcinogenesis. Cancer Res. 2019;7920:5367-81.

11. Fiori ME, et al. Cancer-associated fibroblasts as abettors of tumor progression at the crossroads of EMT and therapy resistance. Mol Cancer. 2019;181:70.

12. Hinshaw DC, Shevde LA. The tumor microenvironment innately modulates cancer progression. Cancer Res. 2019;7918:4557-66.

13. Joshi RS, et al. The role of cancer-associated fibroblasts in tumor progression. Cancers. 2021;136:1399.

14. Martinez-Outschoorn UE, Lisanti MP, Sotgia F. Catabolic cancerassociated energy and biomass to anabolic cancer cells, fueling tumor growth. Semin Cancer Biol. 2014;25:47-60.

15. Kobayashi $\mathrm{H}$, et al. Cancer-associated fibroblasts in gastrointestinal cancer. Nat Rev Gastroenterol Hepatol. 2019;165:282-95.
16. Farhood B, Najafi M, Mortezaee K. Cancer-associated fibroblasts: Secretions, interactions, and therapy. J Cell Biochem. 2019;1203:2791-800.

17. Fullár $A$, et al. Remodeling of extracellular matrix by normal and tumorassociated fibroblasts promotes cervical cancer progression. BMC Cancer. 2015;15:256.

18. Eble JA, Niland S. The extracellular matrix in tumor progression and metastasis. Clin Exp Metastasis. 2019;363:171-98.

19. Chen $Z$, et al. Single-cell RNA sequencing highlights the role of inflammatory cancer-associated fibroblasts in bladder urothelial carcinoma. Nat Commun. 2020;111:5077.

20. Zhang Y, Liu Q, Liao Q. Long noncoding RNA: a dazzling dancer in tumor immune microenvironment. J Exp Clin Cancer Res. 2020;391:231.

21. Lei $X$, et al. Immune cells within the tumor microenvironment: Biological functions and roles in cancer immunotherapy. Cancer Lett. 2020;470:126-33.

22. An Y, Liu F, Chen Y, Yang Q. Crosstalk between cancer-associated fibroblasts and immune cells in cancer. J Cell Mol Med. 2020;241:13-24.

23. Barrett R, Puré E. Cancer-associated fibroblasts: key determinants of tumor immunity and immunotherapy. Curr Opin Immunol. 2020;64:80-7.

24. Barrett RL, Puré E. Cancer-associated fibroblasts and their influence on tumor immunity and immunotherapy. Elife. 2020;9:e57243.

25. Ene-Obong A, et al. Activated pancreatic stellate cells sequester CD8+ T cells to reduce their infiltration of the juxtatumoral compartment of pancreatic ductal adenocarcinoma. Gastroenterology. 2013;1455:1121-32.

26. Zhang $A$, et al. Cancer-associated fibroblasts promote $M 2$ polarization of macrophages in pancreatic ductal adenocarcinoma. Cancer Med. 2017;62:463-70.

27. Kinoshita $T$, et al. Forkhead box $\mathrm{P} 3$ regulatory $T$ cells coexisting with cancer associated fibroblasts are correlated with a poor outcome in lung adenocarcinoma. Cancer Sci. 2013;1044:409-15.

28. Chun E, et al. CCL2 promotes colorectal carcinogenesis by enhancing polymorphonuclear myeloid-derived suppressor cell population and function. Cell Rep. 2015;122:244-57.

29. Erez N, Truitt M, Olson P, Arron ST, Hanahan D. Cancer-associated fibroblasts are activated in incipient neoplasia to orchestrate tumorpromoting inflammation in an NF-kappaB-dependent manner. Cancer Cell. 2010;172:135-47.

30. Kalluri R. The biology and function of fibroblasts in cancer. Nat Rev Cancer. 2016;169:582-98.

31. Louault K, Li RR, DeClerck YA. Cancer-associated fibroblasts: Understanding their heterogeneity. Cancers. 2020;1211:3108.

32. Kretzschmar K, Weber C, Driskell RR, Calonje E, Watt FM. Compartmentalized epidermal activation of $\beta$-catenin differentially affects lineage reprogramming and underlies tumor heterogeneity. Cell Rep. 2016;142:269-81.

33. Arina A, et al. Tumor-associated fibroblasts predominantly come from local and not circulating precursors. Proc Natl Acad Sci U S A. 2016;11327:7551-6.

34. Kuzet SE, Gaggioli C. Fibroblast activation in cancer: when seed fertilizes soil. Cell Tissue Res. 2016:3653:607-19.

35. Hawinkels $L$, et al. Interaction with colon cancer cells hyperactivates TGF- $\beta$ signaling in cancer-associated fibroblasts. Oncogene. 2014;331:97-107.

36. Wu X, et al. Hepatocyte growth factor activates tumor stromal fibroblasts to promote tumorigenesis in gastric cancer. Cancer Lett. 2013;3351:128-35.

37. Elenbaas B, Weinberg RA. Heterotypic signaling between epithelial tumor cells and fibroblasts in carcinoma formation. Exp Cell Res. 2001;2641:169-84.

38. Kojima Y, et al. Autocrine TGF-beta and stromal cell-derived factor-1 (SDF-1) signaling drives the evolution of tumor-promoting mammary stromal myofibroblasts. Proc Natl Acad Sci U S A. 2010;10746:20009-14.

39. Costa A, Scholer-Dahirel A, Mechta-Grigoriou F. The role of reactive oxygen species and metabolism on cancer cells and their microenvironment. Semin Cancer Biol. 2014;25:23-32.

40. Arcucci A, Ruocco MR, Granato G, Sacco AM, Montagnani S. Cancer: An oxidative crosstalk between solid tumor cells and cancer associated fibroblasts. Biomed Res Int. 2016;2016:4502846. 
41. Omary MB, Lugea A, Lowe AW, Pandol SJ. The pancreatic stellate cell: a star on the rise in pancreatic diseases. J Clin Invest. 2007;1171:50-9.

42. Yin C, Evason KJ, Asahina K, Stainier DY. Hepatic stellate cells in liver development, regeneration, and cancer. J Clin Invest. 2013:1235:1902-10.

43. Froeling FE, et al. Retinoic acid-induced pancreatic stellate cell quiescence reduces paracrine Wnt- $\beta$-catenin signaling to slow tumor progression. Gastroenterology. 2011;1414(1486-97):1497.e1-14.

44. Zhou Y, et al. Vitamin A deficiency causes islet dysfunction by inducing islet stellate cell activation via cellular retinol binding protein 1. Int J Biol Sci. 2020;166:947-56.

45. Xie Z, et al. Exosome-delivered CD44v6/C1QBP complex drives pancreatic cancer liver metastasis by promoting fibrotic liver microenvironment. Gut. 2021. https://doi.org/10.1136/gutjnl-2020-323014.

46. Quante M, et al. Bone marrow-derived myofibroblasts contribute to the mesenchymal stem cell niche and promote tumor growth. Cancer Cell. 2011;192:257-72.

47. Coffman LG, et al. Ovarian carcinoma-associated mesenchymal stem cells arise from tissue-specific normal stroma. Stem Cells. 2019:372:257-69.

48. Hashimoto $\mathrm{O}$, et al. Collaboration of cancer-associated fibroblasts and tumour-associated macrophages for neuroblastoma development. J Pathol. 2016;2402:211-23.

49. Suda Y, et al. Clonal heterogeneity in osteogenic potential of lung cancer-associated fibroblasts: promotional effect of osteogenic progenitor cells on cancer cell migration. J Cancer Res Clin Oncol. 2016;1427:1487-98

50. Zhu H, et al. Proton-sensing GPCR-YAP signalling promotes cancerassociated fibroblast activation of mesenchymal stem cells. Int J Biol Sci. 2016;124:389-96.

51. Barcellos-de-Souza P, et al. Mesenchymal Stem Cells are Recruited and Activated into Carcinoma-Associated Fibroblasts by Prostate Cancer Microenvironment-Derived TGF- $\beta 1$. Stem Cells. 2016;3410:2536-47.

52. Jung $Y$, et al. Recruitment of mesenchymal stem cells into prostate tumours promotes metastasis. Nat Commun. 2013:4:1795.

53. Weber CE, et al. Osteopontin mediates an MZF1-TGF- $\beta 1$-dependent transformation of mesenchymal stem cells into cancer-associated fibroblasts in breast cancer. Oncogene. 2015;3437:4821-33.

54. Guido $C$, et al. Metabolic reprogramming of cancer-associated fibroblasts by TGF- $\beta$ drives tumor growth: connecting TGF- $\beta$ signaling with "Warburg-like" cancer metabolism and L-lactate production. Cell Cycle. 2012;1116:3019-35.

55. Shi Y, Du L, Lin L, Wang Y. Tumour-associated mesenchymal stem/ stromal cells: emerging therapeutic targets. Nat Rev Drug Discov. 2017;161:35-52

56. Wang YM, Wang W, Qiu ED. Osteosarcoma cells induce differentiation of mesenchymal stem cells into cancer associated fibroblasts through Notch and Akt signaling pathway. Int J Clin Exp Pathol. 2017;108:8479-86.

57. Peng Y, Li Z, Li Z. GRP78 secreted by tumor cells stimulates differentiation of bone marrow mesenchymal stem cells to cancer-associated fibroblasts. Biochem Biophys Res Commun. 2013;4404:558-63.

58. Bielczyk-Maczynska E. White adipocyte plasticity in physiology and disease. Cells. 2019;812:1507.

59. Zhang $Y$, et al. White adipose tissue cells are recruited by experimental tumors and promote cancer progression in mouse models. Cancer Res. 2009;6912:5259-66.

60. Jotzu C, et al. Adipose tissue derived stem cells differentiate into carcinoma-associated fibroblast-like cells under the influence of tumor derived factors. Cell Oncol. 2011;341:55-67.

61. Bochet $L$, et al. Adipocyte-derived fibroblasts promote tumor progression and contribute to the desmoplastic reaction in breast cancer. Cancer Res. 2013;7318:5657-68.

62. Dirat B, et al. Cancer-associated adipocytes exhibit an activated phenotype and contribute to breast cancer invasion. Cancer Res. 2011;717:2455-65.

63. Rhim AD, et al. EMT and dissemination precede pancreatic tumor formation. Cell. 2012;1481-2:349-61.

64. Fischer KR, et al. Epithelial-to-mesenchymal transition is not required for lung metastasis but contributes to chemoresistance. Nature. 2015;5277579:472-6.
65. Dulauroy S, Di Carlo SE, Langa F, Eberl G, Peduto L. Lineage tracing and genetic ablation of ADAM12(+) perivascular cells identify a major source of profibrotic cells during acute tissue injury. Nat Med. 2012;188:1262-70.

66. Huang $X$, et al. Oxidative stress induces monocyte-to-myofibroblast transdifferentiation through p38 in pancreatic ductal adenocarcinoma. Clin Transl Med. 2020;102:e41.

67. Potenta S, Zeisberg E, Kalluri R. The role of endothelial-to-mesenchymal transition in cancer progression. Br J Cancer. 2008;999:1375-9.

68. Rinkevich Y, et al. Identification and prospective isolation of a mesothelial precursor lineage giving rise to smooth muscle cells and fibroblasts for mammalian internal organs, and their vasculature. Nat Cell Biol. 2012;1412:1251-60.

69. Kalluri R, Weinberg RA. The basics of epithelial-mesenchymal transition. J Clin Invest. 2009;1196:1420-8.

70. Zeisberg EM, Potenta S, Xie L, Zeisberg M, Kalluri R. Discovery of endothelial to mesenchymal transition as a source for carcinomaassociated fibroblasts. Cancer Res. 2007;6721:10123-8.

71. Wei M, et al. Malignant ascites-derived exosomes promote proliferation and induce carcinoma-associated fibroblasts transition in peritoneal mesothelial cells. Oncotarget. 2017;826:42262-71.

72. Nikolic-Paterson DJ, Wang S, Lan HY. Macrophages promote renal fibrosis through direct and indirect mechanisms. Kidney Int Suppl. 2011;2014(41):34-8.

73. Sanz-Moreno V, et al. ROCK and JAK1 signaling cooperate to control actomyosin contractility in tumor cells and stroma. Cancer Cell. 2011;202:229-45.

74. Ershaid N, et al. NLRP3 inflammasome in fibroblasts links tissue damage with inflammation in breast cancer progression and metastasis. Nat Commun. 2019;101:4375.

75. Scherz-Shouval R, et al. The reprogramming of tumor stroma by HSF1 is a potent enabler of malignancy. Cell. 2014;1583:564-78.

76. Ferrari $\mathrm{N}$, et al. Dickkopf-3 links HSF1 and YAP/TAZ signalling to control aggressive behaviours in cancer-associated fibroblasts. Nat Commun. 2019;101:130

77. Albrengues J, et al. Epigenetic switch drives the conversion of fibroblasts into proinvasive cancer-associated fibroblasts. Nat Commun. 2015;6:10204

78. Albrengues J, et al. LIF mediates proinvasive activation of stromal fibroblasts in cancer. Cell Rep. 2014;75:1664-78.

79. Sanchez-Alvarez R, et al. Ethanol exposure induces the cancer-associated fibroblast phenotype and lethal tumor metabolism: implications for breast cancer prevention. Cell Cycle. 2013;122:289-301.

80. Garufi A, Traversi G, Cirone M, D'Orazi G. HIPK2 role in the tumor-host interaction: impact on fibroblasts transdifferentiation CAF-like. IUBMB Life. 2019;7112:2055-61.

81. Calvo F, et al. Mechanotransduction and YAP-dependent matrix remodelling is required for the generation and maintenance of cancerassociated fibroblasts. Nat Cell Biol. 2013;156:637-46.

82. Calvo F, et al. Cdc42EP3/BORG2 and septin network enables mechanotransduction and the emergence of cancer-associated fibroblasts. Cell Rep. 2015;1312:2699-714.

83. Malik R, et al. Rigidity controls human desmoplastic matrix anisotropy to enable pancreatic cancer cell spread via extracellular signal-regulated kinase 2. Matrix Biol. 2019;81:50-69.

84. Avery $D$, et al. Extracellular matrix directs phenotypic heterogeneity of activated fibroblasts. Matrix Biol. 2018;67:90-106.

85. Straub JM, et al. Radiation-induced fibrosis: mechanisms and implications for therapy. J Cancer Res Clin Oncol. 2015;14111:1985-94.

86. Alcolea MP, Jones PH. Tracking cells in their native habitat: lineage tracing in epithelial neoplasia. Nat Rev Cancer. 2013;133:161-71.

87. Sahai $E$, et al. A framework for advancing our understanding of cancerassociated fibroblasts. Nat Rev Cancer. 2020;203:174-86.

88. Ishii G, Ochiai A, Neri S. Phenotypic and functional heterogeneity of cancer-associated fibroblast within the tumor microenvironment. Adv Drug Deliv Rev. 2016;99Pt B:186-96.

89. Moffitt RA, et al. Virtual microdissection identifies distinct tumor- and stroma-specific subtypes of pancreatic ductal adenocarcinoma. Nat Genet. 2015;4710:1168-78.

90. Bailey $\mathrm{P}$, et al. Genomic analyses identify molecular subtypes of pancreatic cancer. Nature. 2016;5317592:47-52.

91. Öhlund $D$, et al. Distinct populations of inflammatory fibroblasts and myofibroblasts in pancreatic cancer. J Exp Med. 2017;2143:579-96. 
92. Elyada E, et al. Cross-species single-cell analysis of pancreatic ductal adenocarcinoma reveals antigen-presenting cancer-associated fibroblasts. Cancer Discov. 2019;98:1102-23.

93. Lakins MA, Ghorani E, Munir H, Martins CP, Shields JD. Cancer-associated fibroblasts induce antigen-specific deletion of CD8 (+) T Cells to protect tumour cells. Nat Commun. 2018;91:948.

94. Hosein AN, et al. Cellular heterogeneity during mouse pancreatic ductal adenocarcinoma progression at single-cell resolution. JCI Insight. 2019:516:e129212.

95. Neuzillet $C$, et al. Inter- and intra-tumoural heterogeneity in cancerassociated fibroblasts of human pancreatic ductal adenocarcinoma. J Pathol. 2019:2481:51-65.

96. Costa A, et al. Fibroblast heterogeneity and immunosuppressive environment in human breast cancer. Cancer Cell. 2018:333:463-479.e10.

97. Givel AM, et al. miR200-regulated CXCL12 $\beta$ promotes fibroblast heterogeneity and immunosuppression in ovarian cancers. Nat Commun. 2018;91:1056

98. Pelon F, et al. Cancer-associated fibroblast heterogeneity in axillary lymph nodes drives metastases in breast cancer through complementary mechanisms. Nat Commun. 2020;111:404.

99. Sebastian A, et al. Single-cell transcriptomic analysis of tumor-derived fibroblasts and normal tissue-resident fibroblasts reveals fibroblast heterogeneity in breast cancer. Cancers. 2020;125:1307.

100. Bartoschek M, et al. Spatially and functionally distinct subclasses of breast cancer-associated fibroblasts revealed by single cell RNA sequencing. Nat Commun. 2018;91:5150.

101. Costea DE, et al. Identification of two distinct carcinoma-associated fibroblast subtypes with differential tumor-promoting abilities in oral squamous cell carcinoma. Cancer Res. 2013;7313:3888-901.

102. Li H, et al. Reference component analysis of single-cell transcriptomes elucidates cellular heterogeneity in human colorectal tumors. Nat Genet. 2017;495:708-18.

103. Jiang $H$, Hegde $S$, DeNardo DG. Tumor-associated fibrosis as a regulator of tumor immunity and response to immunotherapy. Cancer Immunol Immunother. 2017:668:1037-48.

104. Su S, et al. CD10(+)GPR77(+) cancer-associated fibroblasts promote cancer formation and chemoresistance by sustaining cancer stemness. Cell. 2018;1724:841-856.e16.

105. Özdemir BC, et al. Depletion of carcinoma-associated fibroblasts and fibrosis induces immunosuppression and accelerates pancreas cancer with reduced survival. Cancer Cell. 2014;256:719-34.

106. Rhim AD, et al. Stromal elements act to restrain, rather than support, pancreatic ductal adenocarcinoma. Cancer Cell. 2014;256:735-47.

107. Lee JJ, et al. Stromal response to Hedgehog signaling restrains pancreatic cancer progression. Proc Natl Acad Sci U S A. 2014:11130:E3091-100.

108. Patel AK, et al. A subtype of cancer-associated fibroblasts with lower expression of alpha-smooth muscle actin suppresses stemness through BMP4 in oral carcinoma. Oncogenesis. 2018;710:78.

109. Gerling $M$, et al. Stromal Hedgehog signalling is downregulated in colon cancer and its restoration restrains tumour growth. Nat Commun. 2016;7:12321.

110. Shin $\mathrm{K}$, et al. Hedgehog signaling restrains bladder cancer progression by eliciting stromal production of urothelial differentiation factors. Cancer Cell. 2014:264:521-33.

111. Pallangyo CK, Ziegler PK, Greten FR. IKK 3 acts as a tumor suppressor in cancer-associated fibroblasts during intestinal tumorigenesis. J Exp Med. 2015:21213:2253-66.

112. Miyai Y, Esaki N, Takahashi M, Enomoto A. Cancer-associated fibroblasts that restrain cancer progression: Hypotheses and perspectives. Cancer Sci. 2020;1114:1047-57.

113. Kato T, et al. Cancer-associated fibroblasts affect intratumoral CD8(+) and FoxP3(+) T cells via IL6 in the tumor microenvironment. Clin Cancer Res. 2018;2419:4820-33.

114. Feig C, et al. Targeting CXCL12 from FAP-expressing carcinomaassociated fibroblasts synergizes with anti-PD-L1 immunotherapy in pancreatic cancer. Proc Natl Acad Sci U S A. 2013;11050:20212-7.

115. Yoshida GJ. Regulation of heterogeneous cancer-associated fibroblasts: the molecular pathology of activated signaling pathways. J Exp Clin Cancer Res. 2020;391:112.
116. Harper J, Sainson RC. Regulation of the anti-tumour immune response by cancer-associated fibroblasts. Semin Cancer Biol. 2014;25:69-77.

117. Ziani L, Chouaib S, Thiery J. Alteration of the antitumor immune response by cancer-associated fibroblasts. Front Immunol. 2018;9:414

118. Kim R, Emi M, Tanabe K. Cancer immunosuppression and autoimmune disease: beyond immunosuppressive networks for tumour immunity. Immunology. 2006;1192:254-64.

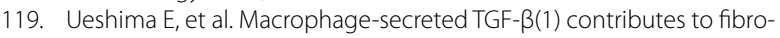
blast activation and ureteral stricture after ablation injury. Am J Physiol Renal Physiol. 2019;3177:F52-f64.

120. Sun $Q$, et al. The impact of cancer-associated fibroblasts on major hallmarks of pancreatic cancer. Theranostics. 2018:818:5072-87.

121. Shiga K, et al. Cancer-associated fibroblasts: their characteristics and their roles in tumor growth. Cancers (Basel). 2015;74:2443-58.

122. Mantovani A, et al. The chemokine system in diverse forms of macrophage activation and polarization. Trends Immunol. 2004;2512:677-86

123. Shapouri-Moghaddam A, et al. Macrophage plasticity, polarization, and function in health and disease. J Cell Physiol. 2018;2339:6425-40.

124. Allavena P, Sica A, Garlanda C, Mantovani A. The Yin-Yang of tumorassociated macrophages in neoplastic progression and immune surveillance. Immunol Rev. 2008;222:155-61.

125. Mantovani A, Allavena P, Sica A, Balkwill F. Cancer-related inflammation. Nature. 2008:4547203:436-44.

126. Hu B, et al. Blockade of DC-SIGN(+) tumor-associated macrophages reactivates antitumor immunity and improves immunotherapy in muscle-invasive bladder cancer. Cancer Res. 2020;808:1707-19.

127. Yugawa $K$, et al. CMTM6 stabilizes PD-L1 expression and is a new prognostic impact factor in hepatocellular carcinoma. Hepatol Commun. 2021;52:334-48.

128. Herrera M, et al. Cancer-associated fibroblast and M2 macrophage markers together predict outcome in colorectal cancer patients. Cancer Sci. 2013;1044:437-44.

129. Fujii N, et al. Cancer-associated fibroblasts and CD163-positive macrophages in oral squamous cell carcinoma: their clinicopathological and prognostic significance. J Oral Pathol Med. 2012;416:444-51.

130. Tan B, et al. Inhibition of Rspo-Lgr4 facilitates checkpoint blockade therapy by switching macrophage polarization. Cancer Res. 2018;7817:4929-42

131. Ksiazkiewicz M, et al. Importance of CCL2-CCR2A/2B signaling for monocyte migration into spheroids of breast cancer-derived fibroblasts. Immunobiology. 2010;2159-10:737-47.

132. Cohen $\mathrm{N}$, et al. Fibroblasts drive an immunosuppressive and growthpromoting microenvironment in breast cancer via secretion of Chitinase 3-like 1. Oncogene. 2017;3631:4457-68.

133. Comito G, et al. Cancer-associated fibroblasts and M2-polarized macrophages synergize during prostate carcinoma progression. Oncogene. 2014;3319:2423-31.

134. Taddei ML, et al. Senescent stroma promotes prostate cancer progression: the role of miR-210. Mol Oncol. 2014;88:1729-46.

135. Mace TA, et al. Pancreatic cancer-associated stellate cells promote differentiation of myeloid-derived suppressor cells in a STAT3-dependent manner. Cancer Res. 2013;7310:3007-18.

136. Nagarsheth N, Wicha MS, Zou W. Chemokines in the cancer microenvironment and their relevance in cancer immunotherapy. Nat Rev Immunol. 2017;179:559-72.

137. Zhang R, et al. Cancer-associated fibroblasts enhance tumor-associated macrophages enrichment and suppress NK cells function in colorectal cancer. Cell Death Dis. 2019:104:273.

138. Zhang J, Chen L, Xiao M, Wang C, Qin Z. FSP1 + fibroblasts promote skin carcinogenesis by maintaining MCP-1-mediated macrophage infiltration and chronic inflammation. Am J Pathol. 2011;1781:382-90.

139. Gordon SR, et al. PD-1 expression by tumour-associated macrophages inhibits phagocytosis and tumour immunity. Nature. 2017:5457655:495-9.

140. Gok Yavuz B, et al. Cancer associated fibroblasts sculpt tumour microenvironment by recruiting monocytes and inducing immunosuppressive PD-1(+) TAMs. Sci Rep. 2019;91:3172.

141. Mazur A, Holthoff E, Vadali S, Kelly T, Post SR. Cleavage of type I collagen by fibroblast activation protein-a enhances class A scavenger receptor mediated macrophage adhesion. PLoS One. 2016;113:e0150287. 
142. Takahashi H, et al. Cancer-associated fibroblasts promote an immunosuppressive microenvironment through the induction and accumulation of protumoral macrophages. Oncotarget. 2017;85:8633-47.

143. Zhang Q, et al. Macrophages activate mesenchymal stem cells to acquire cancer-associated fibroblast-like features resulting in gastric epithelial cell lesions and malignant transformation in vitro. Oncol Lett. 2019;171:747-56.

144. Wu L, Saxena S, Awaji M, Singh RK. Tumor-associated neutrophils in cancer: going pro. Cancers. 2019:114:564

145. Coffelt SB, Wellenstein MD, de Visser KE. Neutrophils in cancer: neutral no more. Nat Rev Cancer. 2016;167:431-46.

146. Fridlender ZG, et al. Polarization of tumor-associated neutrophil phenotype by TGF-beta: "N1" versus "N2"TAN. Cancer Cell. 2009;163:183-94.

147. Fridlender ZG, et al. Transcriptomic analysis comparing tumor-associated neutrophils with granulocytic myeloid-derived suppressor cells and normal neutrophils. PLoS One. 2012;72:e31524.

148. Jablonska J, Leschner S, Westphal K, Lienenklaus S, Weiss S. Neutrophils responsive to endogenous IFN-beta regulate tumor angiogenesis and growth in a mouse tumor model. J Clin Invest. 2010;1204:1151-64.

149. Piccard H, Muschel RJ, Opdenakker G. On the dual roles and polarized phenotypes of neutrophils in tumor development and progression. Crit Rev Oncol Hematol. 2012:823:296-309.

150. Song $\mathrm{M}$, et al. Cancer-associated fibroblast-mediated cellular crosstalk supports hepatocellular carcinoma progression. Hepatology. 2021;735:1717-35.

151. Cheng Y, et al. Cancer-associated fibroblasts induce PDL1+ neutrophils through the IL6-STAT3 pathway that foster immune suppression in hepatocellular carcinoma. Cell Death Dis. 2018;94:422.

152. Fridlender ZG, Albelda SM. Tumor-associated neutrophils: friend or foe? Carcinogenesis. 2012;335:949-55.

153. Raman D, Baugher PJ, Thu YM, Richmond A. Role of chemokines in tumor growth. Cancer Lett. 2007;2562:137-65.

154. Zhu Q, et al. The IL-6-STAT3 axis mediates a reciprocal crosstalk between cancer-derived mesenchymal stem cells and neutrophils to synergistically prompt gastric cancer progression. Cell Death Dis. 2014;56:e1295.

155. Liu J, et al. Mast cell: insight into remodeling a tumor microenvironment. Cancer Metastasis Rev. 2011;302:177-84.

156. Dalton DK, Noelle RJ. The roles of mast cells in anticancer immunity. Cancer Immunol Immunother. 2012;619:1511-20.

157. Derakhshani A, et al. Mast cells: a double-edged sword in cancer. Immunol Lett. 2019:209:28-35.

158. Johansson A, et al. Mast cells are novel independent prognostic markers in prostate cancer and represent a target for therapy. Am J Pathol. 2010;1772:1031-41.

159. Siiskonen $\mathrm{H}$, et al. Low numbers of tryptase+ and chymase+ mast cells associated with reduced survival and advanced tumor stage in melanoma. Melanoma Res. 2015;256:479-85.

160. Hölzel $M$, et al. A preclinical model of malignant peripheral nerve sheath tumor-like melanoma is characterized by infiltrating mast cells. Cancer Res. 2016;762:251-63.

161. Gentles AJ, et al. A human lung tumor microenvironment interactome identifies clinically relevant cell-type cross-talk. Genome Biol. 2020;211:107.

162. Kolset SO, Pejler G. Serglycin: a structural and functional chameleon with wide impact on immune cells. J Immunol. 2011;18710:4927-33.

163. Beer TW, Ng LB, Murray K. Mast cells have prognostic value in Merkel cell carcinoma. Am J Dermatopathol. 2008;301:27-30.

164. Medina $\mathrm{V}$, et al. Histamine-mediated signaling processes in human malignant mammary cells. Cancer Biol Ther. 2006;511:1462-71.

165. Vizio $B$, et al. Pancreatic-carcinoma-cell-derived pro-angiogenic factors can induce endothelial-cell differentiation of a subset of circulating CD34+ progenitors. J Transl Med. 2013;11:314

166. Carmeliet P, Jain RK. Molecular mechanisms and clinical applications of angiogenesis. Nature. 2011;4737347:298-307.

167. Detoraki A, et al. Vascular endothelial growth factors synthesized by human lung mast cells exert angiogenic effects. J Allergy Clin Immunol. 2009;1235(1142-9):1149.e1-5.

168. Baram D, et al. Human mast cells release metalloproteinase-9 on contact with activated T cells: juxtacrine regulation by TNF-alpha. J Immunol. 2001;1677:4008-16.
169. Huang B, et al. SCF-mediated mast cell infiltration and activation exacerbate the inflammation and immunosuppression in tumor microenvironment. Blood. 2008;1124:1269-79.

170. Ribatti D, Crivellato E. Mast cells, angiogenesis and cancer. Adv Exp Med Biol. 2011:716:270-88

171. Maltby S, Khazaie K, McNagny KM. Mast cells in tumor growth: angiogenesis, tissue remodelling and immune-modulation. Biochim Biophys Acta. 2009;17961:19-26.

172. Pereira JDS, et al. Myofibroblasts and mast cells: influences on biological behavior of odontogenic lesions. Ann Diagn Pathol. 2018;34:66-71.

173. Yang FC, et al. Nf1+/- mast cells induce neurofibroma like phenotypes through secreted TGF-beta signaling. Hum Mol Genet. 2006;1516:2421-37

174. Ellem SJ, et al. A pro-tumourigenic loop at the human prostate tumour interface orchestrated by oestrogen, CXCL12 and mast cell recruitment J Pathol. 2014;2341:86-98.

175. Ma Y, Hwang RF, Logsdon CD, Ullrich SE. Dynamic mast cell-stromal cell interactions promote growth of pancreatic cancer. Cancer Res. 2013;7313:3927-37

176. Pereira BA, et al. Tissue engineered human prostate microtissues reveal key role of mast cell-derived tryptase in potentiating cancer-associated fibroblast (CAF)-induced morphometric transition in vitro. Biomaterials. 2019;197:72-85.

177. Guillerey C, Huntington ND, Smyth MJ. Targeting natural killer cells in cancer immunotherapy. Nat Immunol. 2016;179:1025-36.

178. Abel AM, Yang C, Thakar MS, Malarkannan S. Natural killer cells: development, maturation, and clinical utilization. Front Immunol. 2018;9:1869.

179. Chiossone L, Dumas PY, Vienne M, Vivier E. Natural killer cells and other innate lymphoid cells in cancer. Nat Rev Immunol. 2018;1811:671-88.

180. Souza-Fonseca-Guimaraes F, Cursons J, Huntington ND. The emergence of natural killer cells as a major target in cancer immunotherapy. Trends Immunol. 2019:402:142-58.

181. Wang J, Matosevic S. Functional and metabolic targeting of natural killer cells to solid tumors. Cell Oncol (Dordr). 2020;434:577-600.

182. Sivori S, et al. Human NK cells: surface receptors, inhibitory checkpoints, and translational applications. Cell Mol Immunol. 2019;165:430-41.

183. Stojanovic A, Cerwenka A. Natural killer cells and solid tumors. J Innate Immun. 2011;34:355-64.

184. Habif G, Crinier A, André P, Vivier E, Narni-Mancinelli E. Targeting natural killer cells in solid tumors. Cell Mol Immunol. 2019;165:415-22.

185. Turley SJ, Cremasco V, Astarita JL. Immunological hallmarks of stromal cells in the tumour microenvironment. Nat Rev Immunol. 2015;1511:669-82

186. Ziani $L$, et al. Melanoma-associated fibroblasts decrease tumor cell susceptibility to NK cell-mediated killing through matrix-metalloproteinases secretion. Oncotarget. 2017;812:19780-94.

187. Li T, et al. Hepatocellular carcinoma-associated fibroblasts trigger NK cell dysfunction via PGE2 and IDO. Cancer Lett. 2012;3182:154-61.

188. Li T, et al. Colorectal carcinoma-derived fibroblasts modulate natural killer cell phenotype and antitumor cytotoxicity. Med Oncol. 2013;303:663

189. Balsamo M, et al. Melanoma-associated fibroblasts modulate NK cell phenotype and antitumor cytotoxicity. Proc Natl Acad Sci U S A. 2009;10649:20847-52

190. Inoue T, et al. Cancer-associated fibroblast suppresses killing activity of natural killer cells through downregulation of poliovirus receptor (PVR/CD155), a ligand of activating NK receptor. Int J Oncol. 2016:494:1297-304

191. Flavell RA, Sanjabi S, Wrzesinski SH, Licona-Limón P. The polarization of immune cells in the tumour environment by TGFbeta. Nat Rev Immunol. 2010;108:554-67.

192. Batlle $E$, Massagué J. Transforming growth factor- $\beta$ signaling in immunity and cancer. Immunity. 2019:504:924-40.

193. Trotta R, et al. TGF-beta utilizes SMAD3 to inhibit CD16-mediated IFNgamma production and antibody-dependent cellular cytotoxicity in human NK cells. J Immunol. 2008;1816:3784-92.

194. Han B, et al. Altered NKp30, NKp46, NKG2D, and DNAM-1 expression on circulating NK cells is associated with tumor progression in human gastric cancer. J Immunol Res. 2018;2018:6248590. 
195. Donatelli SS, et al. TGF- $\beta$-inducible microRNA-183 silences tumor-associated natural killer cells. Proc Natl Acad Sci U S A. 2014;11111:4203-8.

196. Viel S, et al. TGF- $\beta$ inhibits the activation and functions of NK cells by repressing the mTOR pathway. Sci Signal. 2016;9415:ra19.

197. Lee YS, Radford KJ. The role of dendritic cells in cancer. Int Rev Cell Mol Biol. 2019;348:123-78

198. Wculek SK, et al. Dendritic cells in cancer immunology and immunotherapy. Nat Rev Immunol. 2020;201:7-24.

199. Cheng JT, et al. Hepatic carcinoma-associated fibroblasts induce IDO-producing regulatory dendritic cells through IL-6-mediated STAT3 activation. Oncogenesis. 2016;52:e198.

200. Suciu-Foca N, Berloco P, Cortesini R. Tolerogenic dendritic cells in cancer, transplantation, and autoimmune diseases. Hum Immunol. 2009;705:277-80.

201. Kuo PL, et al. Lung cancer-derived galectin-1 mediates dendritic cell anergy through inhibitor of DNA binding 3/IL-10 signaling pathway. J Immunol. 2011;1863:1521-30.

202. Hsu YL, et al. Lung cancer-derived galectin-1 contributes to cancer associated fibroblast-mediated cancer progression and immune suppression through TDO2/kynurenine axis. Oncotarget. 2016;719:27584-98.

203. Oyama T, et al. Vascular endothelial growth factor affects dendritic cell maturation through the inhibition of nuclear factor-kB activation in hemopoietic progenitor cells. J Immunol. 1998;1603:1224-32.

204. Rahma OE, Hodi FS. The intersection between tumor angiogenesis and immune suppression. Clin Cancer Res. 2019;2518:5449-57.

205. Curiel TJ, et al. Blockade of B7-H1 improves myeloid dendritic cellmediated antitumor immunity. Nat Med. 2003;95:562-7.

206. Kumar BV, Connors TJ, Farber DL. Human T cell development, localization, and function throughout life. Immunity. 2018;482:202-13.

207. Tanaka A, Sakaguchi S. Regulatory T cells in cancer immunotherapy. Cell Res. 2017;271:109-18.

208. Jacobs J, et al. Unveiling a CD70-positive subset of cancer-associated fibroblasts marked by pro-migratory activity and thriving regulatory $T$ cell accumulation. Oncoimmunology. 2018;77:e1440167.

209. Karnoub AE, et al. Mesenchymal stem cells within tumour stroma promote breast cancer metastasis. Nature. 2007;4497162:557-63.

210. Tan W, et al. Tumour-infiltrating regulatory T cells stimulate mammary cancer metastasis through RANKL-RANK signalling. Nature. 2011:4707335:548-53.

211. Bourhis M, Palle J, Galy-Fauroux I, Terme M. Direct and indirect modulation of T cells by VEGF-A counteracted by anti-angiogenic treatment. Front Immunol. 2021;12:616837.

212. Wada J, et al. The contribution of vascular endothelial growth factor to the induction of regulatory T-cells in malignant effusions. Anticancer Res. 2009;293:881-8.

213. Chen W, et al. Conversion of peripheral CD4+CD25- naive T cells to CD4+CD25+ regulatory $T$ cells by TGF-beta induction of transcription factor Foxp3. J Exp Med. 2003;19812:1875-86.

214. Zhao X, et al. Diminished CD68(+) cancer-associated fibroblast subset induces regulatory T-Cell (Treg) infiltration and predicts poor prognosis of oral squamous cell carcinoma patients. Am J Pathol. 2020:1904:886-99.

215. Zhu J.THelper cell differentiation, heterogeneity, and plasticity. Cold Spring Harb Perspect Biol. 2018;1010:a030338.

216. Zhang Y, Zhang Y, Gu W, He L, Sun B. Th1/Th2 cell's function in immune system. Adv Exp Med Biol. 2014;841:45-65.

217. Liao D, Luo Y, Markowitz D, Xiang R, Reisfeld RA. Cancer associated fibroblasts promote tumor growth and metastasis by modulating the tumor immune microenvironment in a 4T1 murine breast cancer model. PLoS One. 2009;411:e7965.

218. De Monte L, et al. Intratumor T helper type 2 cell infiltrate correlates with cancer-associated fibroblast thymic stromal lymphopoietin production and reduced survival in pancreatic cancer. J Exp Med. 2011;2083:469-78.

219. Comito G, et al. Lactate modulates CD4(+) T-cell polarization and induces an immunosuppressive environment, which sustains prostate carcinoma progression via TLR8/miR21 axis. Oncogene. 2019:3819:3681-95.

220. Gutcher I, et al. Autocrine transforming growth factor- $\beta 1$ promotes in vivo Th17 cell differentiation. Immunity. 2011;343:396-408.
221. Farhood B, Najafi M, Mortezaee K. CD8(+) cytotoxic T lymphocytes in cancer immunotherapy: a review. J Cell Physiol. 2019;2346:8509-21.

222. Uzhachenko RV, Shanker A. CD8(+) T lymphocyte and NK cell network: circuitry in the cytotoxic domain of immunity. Front Immunol. 2019;10:1906.

223. Freeman P, Mielgo A. Cancer-associated fibroblast mediated inhibition of CD8+ cytotoxic T cell accumulation in tumours: mechanisms and therapeutic opportunities. Cancers. 2020;129:2687.

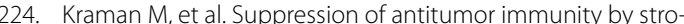
mal cells expressing fibroblast activation protein-alpha. Science. 2010;3306005:827-30

225. Yang $X$, et al. FAP promotes immunosuppression by cancer-associated fibroblasts in the tumor microenvironment via STAT3-CCL2 signaling. Cancer Res. 2016;7614:4124-35.

226. Henke E, Nandigama R, Ergün S. Extracellular matrix in the tumor microenvironment and its impact on cancer therapy. Front Mol Biosci. 2019;6:160.

227. De Francesco EM, et al. HIF-1a/GPER signaling mediates the expression of VEGF induced by hypoxia in breast cancer associated fibroblasts (CAFs). Breast Cancer Res. 2013;154:R64.

228. Bellone M, Calcinotto A. Ways to enhance lymphocyte trafficking into tumors and fitness of tumor infiltrating lymphocytes. Front Oncol. 2013;3:231.

229. Thomas DA, Massagué J. TGF-beta directly targets cytotoxic T cell functions during tumor evasion of immune surveillance. Cancer Cell. 2005;85:369-80

230. Goehrig D, et al. Stromal protein ßig-h3 reprogrammes tumour microenvironment in pancreatic cancer. Gut. 2019;684:693-707.

231. Ino Y, et al. Arginase II expressed in cancer-associated fibroblasts indicates tissue hypoxia and predicts poor outcome in patients with pancreatic cancer. PLoS One. 2013;82:e55146.

232. Rabinovich GA, Toscano MA. Turning 'sweet' on immunity: galectinglycan interactions in immune tolerance and inflammation. Nat Rev Immunol. 2009:95:338-52.

233. Valach J, et al. Smooth muscle actin-expressing stromal fibroblasts in head and neck squamous cell carcinoma: increased expression of galectin-1 and induction of poor prognosis factors. Int J Cancer. 2012;13111:2499-508

234. Gabrilovich DI. Myeloid-derived suppressor cells. Cancer Immunol Res. 2017:51:3-8.

235. Talmadge JE, Gabrilovich DI. History of myeloid-derived suppressor cells. Nat Rev Cancer. 2013;1310:739-52.

236. Ugel S, De Sanctis F, Mandruzzato S, Bronte V. Tumor-induced myeloid deviation: when myeloid-derived suppressor cells meet tumor-associated macrophages. J Clin Invest. 2015:1259:3365-76.

237. Youn JI, Collazo M, Shalova IN, Biswas SK, Gabrilovich DI. Characterization of the nature of granulocytic myeloid-derived suppressor cells in tumor-bearing mice. J Leukoc Biol. 2012;911:167-81.

238. Marvel D, Gabrilovich DI. Myeloid-derived suppressor cells in the tumor microenvironment: expect the unexpected. J Clin Invest. 2015;1259:3356-64.

239. Condamine T, Ramachandran I, Youn Jl, Gabrilovich DI. Regulation of tumor metastasis by myeloid-derived suppressor cells. Annu Rev Med. 2015:66:97-110.

240. Gunaydin G, Kesikli SA, Guc D. Cancer associated fibroblasts have phenotypic and functional characteristics similar to the fibrocytes that represent a novel MDSC subset. Oncoimmunology. 2015;49:e1034918.

241. Qian BZ, et al. CCL2 recruits inflammatory monocytes to facilitate breast-tumour metastasis. Nature. 2011;4757355:222-5.

242. Xiang $\mathrm{H}$, et al. Cancer-associated fibroblasts promote immunosuppression by inducing ROS-generating monocytic MDSCs in lung squamous cell carcinoma. Cancer Immunol Res. 2020;84:436-50.

243. Deng $Y$, et al. Hepatic carcinoma-associated fibroblasts enhance immune suppression by facilitating the generation of myeloid-derived suppressor cells. Oncogene. 2017:368:1090-101.

244. Allaoui R, et al. Cancer-associated fibroblast-secreted CXCL16 attracts monocytes to promote stroma activation in triple-negative breast cancers. Nat Commun. 2016;7:13050.

245. Zhao $\mathrm{Q}$ et al. Cancer-associated fibroblasts induce monocytic myeloidderived suppressor cell generation via IL-6/exosomal miR-21-activated STAT3 signaling to promote cisplatin resistance in esophageal squamous cell carcinoma. Cancer Lett. 2021. 
246. Ohshio Y, Hanaoka J, Kontani K, Teramoto K. Tranilast inhibits the function of cancer-associated fibroblasts responsible for the induction of immune suppressor cell types. Scand I Immunol. 2014;806:408-16.

247. Kumar V, et al. Cancer-associated fibroblasts neutralize the anti-tumor effect of CSF1 receptor blockade by inducing PMN-MDSC infiltration of tumors. Cancer Cell. 2017;325:654-668.e5.

248. Thommen DS, Schumacher TN. T cell dysfunction in cancer. Cancer Cell. 2018;334:547-62

249. Thommen DS, et al. Progression of lung cancer is associated with increased dysfunction of T cells defined by coexpression of multiple inhibitory receptors. Cancer Immunol Res. 2015;312:1344-55.

250. Li J, et al. Tumor-infiltrating Tim-3(+) T cells proliferate avidly except when PD-1 is co-expressed: evidence for intracellular cross talk. Oncoimmunology. 2016;510:e1200778.

251. LuX, et al. Tumor antigen-specific CD8(+) T cells are negatively regulated by PD-1 and Tim-3 in human gastric cancer. Cell Immunol. 2017:313:43-51

252. Sun C, Mezzadra R, Schumacher TN. Regulation and function of the PD-L1 checkpoint. Immunity. 2018;483:434-52.

253. Pearson MJ, et al. Endogenous galectin-9 suppresses apoptosis in human rheumatoid arthritis synovial fibroblasts. Sci Rep. 2018;81:12887.

254. Curran TA, Jalili RB, Farrokhi A, Ghahary A. IDO expressing fibroblasts promote the expansion of antigen specific regulatory T cells. Immunobiology. 2014:2191:17-24.

255. Zhan $\mathrm{S}$, et al. Overexpression of B7-H3 in a-SMA-positive fibroblasts is associated with cancer progression and survival in gastric adenocarcinomas. Front Oncol. 2019;9:1466

256. Li Q, et al. The combined expressions of B7H4 and ACOT4 in cancerassociated fibroblasts are related to poor prognosis in patients with gastric carcinoma. Int J Clin Exp Pathol. 2019;127:2672-81.

257. Khalili JS, et al. Oncogenic BRAF(V600E) promotes stromal cell-mediated immunosuppression via induction of interleukin-1 in melanoma. Clin Cancer Res. 2012;1819:5329-40.

258. Nazareth MR, et al. Characterization of human lung tumor-associated fibroblasts and their ability to modulate the activation of tumor-associated T cells. J Immunol. 2007;1789:5552-62.

259. Pinchuk IV, et al. PD-1 ligand expression by human colonic myofibroblasts/fibroblasts regulates CD4+ T-cell activity. Gastroenterology. 2008;1354(1228-1237):1237.e1-2.

260. Zhang S, Zhou C, Zhang D, Huang Z, Zhang G. The anti-apoptotic effect on cancer-associated fibroblasts of B7-H3 molecule enhancing the cell invasion and metastasis in renal cancer. Onco Targets Ther 2019;12:4119-27.

261. Gorchs $L$, et al. Human pancreatic carcinoma-associated fibroblasts promote expression of co-inhibitory markers on $\mathrm{CD} 4(+)$ and $\mathrm{CD} 8(+)$ T-Cells. Front Immunol. 2019:10:847.

262. Inoue C, et al. PD-L1 induction by cancer-associated fibroblast-derived factors in lung adenocarcinoma cells. Cancers. 2019;1 19:1257.

263. Li Z, et al. Cancer-associated fibroblasts promote PD-L1 expression in mice cancer cells via secreting CXCL5. Int J Cancer. 2019;1457:1946-57.

264. Zhang M, Shi R, Guo Z, He J. Cancer-associated fibroblasts promote cell growth by activating ERK5/PD-L1 signaling axis in colorectal cancer. Pathol Res Pract. 2020;2164:152884.

265. Dou D, et al. Cancer-associated fibroblasts-derived exosomes suppress immune cell function in breast cancer via the miR-92/PD-L1 pathway. Front Immunol. 2020;11:2026.

266. Ozbek S, Balasubramanian PG, Chiquet-Ehrismann R, Tucker RP, Adams JC. The evolution of extracellular matrix. Mol Biol Cell. 2010;2124:4300-5.

267. Malandrino A, Mak M, Kamm RD, Moeendarbary E. Complex mechanics of the heterogeneous extracellular matrix in cancer. Extreme Mech Lett. 2018:21:25-34.

268. Levental KR, et al. Matrix crosslinking forces tumor progression by enhancing integrin signaling. Cell. 2009;1395:891-906.

269. Liu T, Zhou L, Li D, Andl T, Zhang Y. Cancer-associated fibroblasts build and secure the tumor microenvironment. Front Cell Dev Biol. 2019;7:60

270. Erdogan B, Webb DJ. Cancer-associated fibroblasts modulate growth factor signaling and extracellular matrix remodeling to regulate tumor metastasis. Biochem Soc Trans. 2017:451:229-36.

271. Miles FL, Sikes RA. Insidious changes in stromal matrix fuel cancer progression. Mol Cancer Res. 2014;123:297-312.
272. Sato T, et al. Identification of an active site of EMMPRIN for the augmentation of matrix metalloproteinase- 1 and -3 expression in a co-culture of human uterine cervical carcinoma cells and fibroblasts. Gynecol Oncol. 2009;1142:337-42.

273. Murphy G, Nagase H. Progress in matrix metalloproteinase research. Mol Aspects Med. 2008;295:290-308.

274. Lopez II, Kang I, You WK, McDonald DM, Weaver VM. In situ force mapping of mammary gland transformation. Integr Biol (Camb). 2011;39:910-21.

275. Casey TM, et al. Cancer associated fibroblasts stimulated by transforming growth factor beta1 (TGF-beta 1) increase invasion rate of tumor cells: a population study. Breast Cancer Res Treat. 2008;1101:39-49.

276. Chakravarthy A, Khan L, Bensler NP, Bose P, De Carvalho DD. TGF- $\beta$ associated extracellular matrix genes link cancer-associated fibroblasts to immune evasion and immunotherapy failure. Nat Commun. 2018;91:4692

277. Truffi M, Sorrentino L, Corsi F. Fibroblasts in the tumor microenvironment. Adv Exp Med Biol. 2020;1234:15-29.

278. Acerbi I, et al. Human breast cancer invasion and aggression correlates with ECM stiffening and immune cell infiltration. Integr Biol (Camb). 2015;710:1120-34.

279. Sorokin L. The impact of the extracellular matrix on inflammation. Nat Rev Immunol. 2010;1010:712-23.

280. Joyce JA, Fearon DT. T cell exclusion, immune privilege, and the tumor microenvironment. Science. 2015;3486230:74-80.

281. Salmon $\mathrm{H}$, et al. Matrix architecture defines the preferential localization and migration of T cells into the stroma of human lung tumors. J Clin Invest. 2012;1223:899-910.

282. Hartmann N, et al. Prevailing role of contact guidance in intrastromal T-cell trapping in human pancreatic cancer. Clin Cancer Res. 2014;2013:3422-33.

283. Gilkes DM, Semenza GL, Wirtz D. Hypoxia and the extracellular matrix: drivers of tumour metastasis. Nat Rev Cancer. 2014;146:430-9.

284. Jiang $\mathrm{H}$, et al. Targeting focal adhesion kinase renders pancreatic cancers responsive to checkpoint immunotherapy. Nat Med. 2016;228:851-60

285. Diop-Frimpong B, Chauhan VP, Krane S, Boucher Y, Jain RK. Losartan inhibits collagen I synthesis and improves the distribution and efficacy of nanotherapeutics in tumors. Proc Natl Acad Sci U S A. 2011;1087:2909-14.

286. Jiang $\mathrm{H}$, et al. Development of resistance to FAK inhibition in pancreatic cancer is linked to stromal depletion. Gut. 2020:691:122-32.

287. Varol C. Tumorigenic interplay between macrophages and collagenous matrix in the tumor microenvironment. Methods Mol Biol. 2019;1944:203-20.

288. Van Goethem E, Poincloux R, Gauffre F, Maridonneau-Parini I, Le Cabec $V$. Matrix architecture dictates three-dimensional migration modes of human macrophages: differential involvement of proteases and podosome-like structures. J Immunol. 2010;1842:1049-61.

289. McWhorter FY, Wang T, Nguyen P, Chung T, Liu WF. Modulation of macrophage phenotype by cell shape. Proc Natl Acad Sci U S A. 2013;11043:17253-8.

290. Stahl M, et al. Lung collagens perpetuate pulmonary fibrosis via CD204 and M2 macrophage activation. PLoS One. 2013;811:e81382.

291. Patel NR, et al. Cell elasticity determines macrophage function. PLoS One. 2012;79:e41024.

292. Serrels A, et al. Nuclear FAK controls chemokine transcription, tregs, and evasion of anti-tumor immunity. Cell. 2015;1631:160-73.

293. Bae YH, et al. A FAK-Cas-Rac-lamellipodin signaling module transduces extracellular matrix stiffness into mechanosensitive cell cycling. Sci Signal. 2014;7330:ra57.

294. Darvin P, Toor SM, Sasidharan Nair V, Elkord E. Immune checkpoint inhibitors: recent progress and potential biomarkers. Exp Mol Med. 2018;5012:1-11.

295. Duperret EK, et al. Alteration of the tumor stroma using a consensus DNA vaccine targeting fibroblast activation protein (FAP) synergizes with antitumor vaccine therapy in mice. Clin Cancer Res. 2018:245:1190-201.

296. Xia Q, et al. Improvement of anti-tumor immunity of fibroblast activation protein a based vaccines by combination with cyclophosphamide in a murine model of breast cancer. Cell Immunol. 2016:310:89-98. 
297. Xia Q, et al. Cyclophosphamide enhances anti-tumor effects of a fibroblast activation protein a-based DNA vaccine in tumor-bearing mice with murine breast carcinoma. Immunopharmacol Immunotoxicol. 2017;391:37-44

298. Gottschalk S, Yu F, Ji M, Kakarla S, Song XT. A vaccine that co-targets tumor cells and cancer associated fibroblasts results in enhanced antitumor activity by inducing antigen spreading. PLoS One. 2013;812:e82658.

299. Ohshio Y, et al. Cancer-associated fibroblast-targeted strategy enhances antitumor immune responses in dendritic cell-based vaccine. Cancer Sci. 2015;1062:134-42.

300. Zhang Y, Ertl HC. Depletion of FAP + cells reduces immunosuppressive cells and improves metabolism and functions CD8+T cells within tumors. Oncotarget. 2016:717:23282-99.

301. Lo A, et al. Tumor-promoting desmoplasia is disrupted by depleting FAP-expressing stromal cells. Cancer Res. 2015;7514:2800-10.

302. Brennen WN, Rosen DM, Wang H, Isaacs JT, Denmeade SR. Targeting carcinoma-associated fibroblasts within the tumor stroma with a fibroblast activation protein-activated prodrug. J Natl Cancer Inst. 2012;10417:1320-34.

303. Narra K, et al. Phase II trial of single agent Val-boroPro (Talabostat) inhibiting fibroblast activation protein in patients with metastatic colorectal cancer. Cancer Biol Ther. 2007;611:1691-9.

304. Eager RM, et al. Phase Il assessment of talabostat and cisplatin in second-line stage IV melanoma. BMC Cancer. 2009;9:263.

305. Brünker P, et al. RG7386, a novel tetravalent FAP-DR5 antibody, effectively triggers FAP-dependent, avidity-driven DR5 hyperclustering and tumor cell apoptosis. Mol Cancer Ther. 2016;155:946-57.

306. Fang J, et al. A potent immunotoxin targeting fibroblast activation protein for treatment of breast cancer in mice. Int J Cancer. 2016;1384:1013-23.

307. Fang J, et al. A multi-antigen vaccine in combination with an immunotoxin targeting tumor-associated fibroblast for treating murine melanoma. Mol Ther Oncolytics. 2016;3:16007.

308. Tansi FL, et al. Activatable bispecific liposomes bearing fibroblast activation protein directed single chain fragment/Trastuzumab deliver encapsulated cargo into the nuclei of tumor cells and the tumor microenvironment simultaneously. Acta Biomater. 2017;54:281-93.

309. Murakami M, et al. Docetaxel conjugate nanoparticles that target a-smooth muscle actin-expressing stromal cells suppress breast cancer metastasis. Cancer Res. 2013;7315:4862-71.

310. Özdemir BC, et al. Depletion of carcinoma-associated fibroblasts and fibrosis induces immunosuppression and accelerates pancreas cancer with reduced survival. Cancer Cell. 2015;286:831-3.

311. Randomized trial of crenolanib in subjects with D842V mutated GIST. 2021. US National Library of Medicine. ClinicalTrials.gov. https://clinicaltr ials.gov/ct2/show/NCT02847429. Accessed 202122 Jan.

312. Chen $\mathrm{M}$, et al. A whole-cell tumor vaccine modified to express fibroblast activation protein induces antitumor immunity against both tumor cells and cancer-associated fibroblasts. Sci Rep. 2015;5:14421

313. Faivre $\mathrm{S}$, et al. Novel transforming growth factor beta receptor I kinase inhibitor galunisertib (LY2157299) in advanced hepatocellular carcinoma. Liver Int. 2019;398:1468-77.

314. Melisi D, et al. Galunisertib plus gemcitabine vs. gemcitabine for firstline treatment of patients with unresectable pancreatic cancer. $\mathrm{Br} J$ Cancer. 2018;11910:1208-14.

315. Xia Q, et al. Anti-tumor effects of DNA vaccine targeting human fibroblast activation protein a by producing specific immune responses and altering tumor microenvironment in the 4T1 murine breast cancer model. Cancer Immunol Immunother. 2016;655:613-24.

316. Dijkgraaf EM, et al. A phase I trial combining carboplatin/doxorubicin with tocilizumab, an anti-IL-6R monoclonal antibody, and interferon$\mathrm{a} 2 \mathrm{~b}$ in patients with recurrent epithelial ovarian cancer. Ann Oncol. 2015;2610:2141-9.

317. Linehan D, et al. Overall survival in a trial of orally administered CCR2 inhibitor CCX872 in locally advanced/metastatic pancreatic cancer: Correlation with blood monocyte counts. J Clin Oncol. 2018:36(5_suppl):92-92.

318. Noel MS, et al. Orally administered CCR2 selective inhibitor CCX872-b clinical trial in pancreatic cancer. J Clin Oncol. 2017;35(4_suppl):276-276.
319. Mårlind J, et al. Antibody-mediated delivery of interleukin-2 to the stroma of breast cancer strongly enhances the potency of chemotherapy. Clin Cancer Res. 2008;1420:6515-24.

320. Study of FAK (Defactinib) and PD-1 (Pembrolizumab) inhibition in advanced solid malignancies (FAK-PD1). 2018. US National Library of Medicine. ClinicalTrials.gov. https://clinicaltrials.gov/ct2/show/NCT02 758587. Accessed $201819 \mathrm{Mar}$.

321. Rolle $\mathrm{K}$, et al. Promising human brain tumors therapy with interference RNA intervention (iRNAi). Cancer Biol Ther. 2010;95:396-406.

322. Doherty GJ, Tempero M, Corrie PG. HALO-109-301: a Phase III trial of PEGPH20 (with gemcitabine and nab-paclitaxel) in hyaluronic acid-high stage IV pancreatic cancer. Future Oncol. 2018;141:13-22.

323. Chauhan VP, et al. Angiotensin inhibition enhances drug delivery and potentiates chemotherapy by decompressing tumour blood vessels. Nat Commun. 2013;4:2516.

324. Nurmik M, Ullmann P, Rodriguez F, Haan S, Letellier E. In search of definitions: cancer-associated fibroblasts and their markers. Int J Cancer 2020;1464:895-905.

325. Jiang $G M$, et al. The application of the fibroblast activation protein a-targeted immunotherapy strategy. Oncotarget. 2016;722:33472-82.

326. Wang LC, et al. Targeting fibroblast activation protein in tumor stroma with chimeric antigen receptor $T$ cells can inhibit tumor growth and augment host immunity without severe toxicity. Cancer Immunol Res. 2014;22:154-66.

327. Lee J, Fassnacht M, Nair S, Boczkowski D, Gilboa E. Tumor immunotherapy targeting fibroblast activation protein, a product expressed in tumor-associated fibroblasts. Cancer Res. 2005:6523:11156-63.

328. Ostermann E, et al. Effective immunoconjugate therapy in cancer models targeting a serine protease of tumor fibroblasts. Clin Cancer Res. 2008;1414:4584-92.

329. Puré $\mathrm{E}$, Blomberg R. Pro-tumorigenic roles of fibroblast activation protein in cancer: back to the basics. Oncogene. 2018;3732:4343-57.

330. Loeffler M, Krüger JA, Niethammer AG, Reisfeld RA. Targeting tumorassociated fibroblasts improves cancer chemotherapy by increasing intratumoral drug uptake. J Clin Invest. 2006;1167:1955-62.

331. McNutt M. Cancer immunotherapy. Science. 2013;3426165:1417.

332. Santos PM, Butterfield LH. Dendritic cell-based cancer vaccines. J Immunol. 2002;2018:443-9.

333. Song XT, et al. A20 is an antigen presentation attenuator, and its inhibition overcomes regulatory T cell-mediated suppression. Nat Med. 2008;143:258-65.

334. Qian L, et al. Fusion of dendritic cells and cancer-associated fibroblasts for activation of anti-tumor cytotoxic T lymphocytes. J Biomed Nanotechnol. 2018;1410:1826-35.

335. Xia Q, et al. Enhancement of fibroblast activation protein a-based vaccines and adenovirus boost immunity by cyclophosphamide through inhibiting IL-10 expression in 4T1 tumor bearing mice. Vaccine. 2016:3438:4526-35.

336. Meng $\mathrm{M}$, et al. Immunization of stromal cell targeting fibroblast activation protein providing immunotherapy to breast cancer mouse model. Tumour Biol. 2016;378:10317-27.

337. Jiang GM, et al. Curcumin combined with FAPac vaccine elicits effective antitumor response by targeting indolamine-2,3-dioxygenase and inhibiting EMT induced by TNF-a in melanoma. Oncotarget. 2015;628:25932-42.

338. Schuberth PC, et al. Treatment of malignant pleural mesothelioma by fibroblast activation protein-specific re-directed T cells. J Transl Med. 2013:11:187.

339. Roberts EW, et al. Depletion of stromal cells expressing fibroblast activation protein-a from skeletal muscle and bone marrow results in cachexia and anemia. J Exp Med. 2013;2106:1137-51.

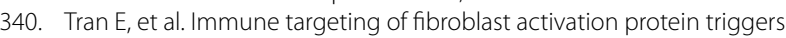
recognition of multipotent bone marrow stromal cells and cachexia. J Exp Med. 2013;2106:1125-35.

341. Deng $L J$, et al. Fibroblast activation protein a activated tripeptide bufadienolide antitumor prodrug with reduced cardiotoxicity. J Med Chem. 2017:6013:5320-33.

342. Brennen WN, et al. Pharmacokinetics and toxicology of a fibroblast activation protein (FAP)-activated prodrug in murine xenograft models of human cancer. Prostate. 2014;7413:1308-19.

343. Rabenhold M, Steiniger F, Fahr A, Kontermann RE, Rüger R. Bispecific single-chain diabody-immunoliposomes targeting endoglin (CD105) 
and fibroblast activation protein (FAP) simultaneously. J Control Release. 2015;201:56-67.

344. Haubeiss S, et al. Dasatinib reverses cancer-associated fibroblasts (CAFs) from primary lung carcinomas to a phenotype comparable to that of normal fibroblasts. Mol Cancer. 2010;9:168.

345. Kovacs RJ, et al. Cardiac safety of TGF- $\beta$ receptor I kinase inhibitor LY2157299 monohydrate in cancer patients in a first-in-human dose study. Cardiovasc Toxicol. 2015;154:309-23.

346. Mariathasan S, et al. TGF $\beta$ attenuates tumour response to PD-L1 blockade by contributing to exclusion of T cells. Nature. 2018;5547693:544-8.

347. Wei Y, et al. Fibroblast-specific inhibition of TGF- $\beta 1$ signaling attenuates lung and tumor fibrosis. J Clin Invest. 2017;12710:3675-88.

348. Holmgaard RB, et al. Targeting the TGF $\beta$ pathway with galunisertib, a TGF $\beta R I$ small molecule inhibitor, promotes anti-tumor immunity leading to durable, complete responses, as monotherapy and in combination with checkpoint blockade. J Immunother Cancer. 2018;61:47.

349. Ravi R, et al. Bifunctional immune checkpoint-targeted antibody-ligand traps that simultaneously disable TGF $\beta$ enhance the efficacy of cancer immunotherapy. Nat Commun. 2018;91:741.

350. Heichler C, et al. STAT3 activation through IL-6/L-11 in cancerassociated fibroblasts promotes colorectal tumour development and correlates with poor prognosis. Gut. 2020;697:1269-82.

351. Yu H, Lee H, Herrmann A, Buettner R, Jove R. Revisiting STAT3 signalling in cancer: new and unexpected biological functions. Nat Rev Cancer. 2014;1411:736-46.

352. Johnson DE, O'Keefe RA, Grandis JR. Targeting the IL-6/JAK/STAT3 signalling axis in cancer. Nat Rev Clin Oncol. 2018;154:234-48.

353. Ham IH, et al. Targeting interleukin- 6 as a strategy to overcome stromainduced resistance to chemotherapy in gastric cancer. Mol Cancer. 2019;181:68.

354. Yanaihara N, et al. Antitumor effects of interleukin-6 (IL-6)/interleukin-6 receptor (IL-6R) signaling pathway inhibition in clear cell carcinoma of the ovary. Mol Carcinog. 2016;555:832-41.

355. Goumas FA, et al. Inhibition of IL-6 signaling significantly reduces primary tumor growth and recurrencies in orthotopic xenograft models of pancreatic cancer. Int J Cancer. 2015;1375:1035-46.

356. Liu H, Shen J, Lu K. IL-6 and PD-L1 blockade combination inhibits hepatocellular carcinoma cancer development in mouse model. Biochem Biophys Res Commun. 2017;4862:239-44.
357. Mace TA, et al. IL-6 and PD-L1 antibody blockade combination therapy reduces tumour progression in murine models of pancreatic cancer. Gut. 2018;672:320-32.

358. Nywening TM, et al. Targeting tumour-associated macrophages with CCR2 inhibition in combination with FOLFIRINOX in patients with borderline resectable and locally advanced pancreatic cancer: a single-centre, open-label, dose-finding, non-randomised, phase $1 \mathrm{~b}$ trial. Lancet Oncol. 2016;175:651-62.

359. McCarthy JB, El-Ashry D, Turley EA. Hyaluronan, cancer-associated fibroblasts and the tumor microenvironment in malignant progression. Front Cell Dev Biol. 2018;6:48.

360. Spenlé $C$, et al. Tenascin- $C$ : Exploitation and collateral damage in cancer management. Cell Adh Migr. 2015;91-2:141-53.

361. Brack SS, Silacci M, Birchler M, Neri D. Tumor-targeting properties of novel antibodies specific to the large isoform of tenascin-C. Clin Cancer Res. 2006;1210:3200-8.

362. Li ZL, et al. Autophagy deficiency promotes triple-negative breast cancer resistance to $\mathrm{T}$ cell-mediated cytotoxicity by blocking tenascin- $\mathrm{C}$ degradation. Nat Commun. 2020;111:3806.

363. Provenzano PP, et al. Enzymatic targeting of the stroma ablates physical barriers to treatment of pancreatic ductal adenocarcinoma. Cancer Cell. 2012;213:418-29.

364. Vandenbroucke RE, Libert C. Is there new hope for therapeutic matrix metalloproteinase inhibition? Nat Rev Drug Discov. 2014;1312:904-27.

365. Franco-Barraza J, et al. Matrix-regulated integrin a(v) $\beta(5)$ maintains $a(5)$ $\beta(1)$-dependent desmoplastic traits prognostic of neoplastic recurrence. Elife. 2017;6:e20600.

366. Ping Q, et al. Cancer-associated fibroblasts: overview, progress, challenges, and directions. Cancer Gene Ther. 2021;28(9):984-99.

367. Elwakeel $\mathrm{E}$, et al. Phenotypic plasticity of fibroblasts during mammary carcinoma development. Int J Mol Sci. 2019;2018:4438.

\section{Publisher's Note}

Springer Nature remains neutral with regard to jurisdictional claims in published maps and institutional affiliations.
Ready to submit your research? Choose BMC and benefit from:

- fast, convenient online submission

- thorough peer review by experienced researchers in your field

- rapid publication on acceptance

- support for research data, including large and complex data types

- gold Open Access which fosters wider collaboration and increased citations

- maximum visibility for your research: over $100 \mathrm{M}$ website views per year

At BMC, research is always in progress.

Learn more biomedcentral.com/submissions 